Review

\title{
Alginate hydrogels for bone tissue engineering, from injectables to bioprinting: A review
}

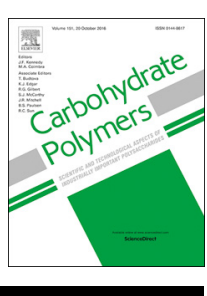

\author{
Aurora C. Hernández-González ${ }^{\mathrm{a}, *}$, Lucía Téllez-Jurado ${ }^{\text {a }}$ Luis M. Rodríguez-Lorenzo ${ }^{\mathrm{b}, \mathrm{c}}$ \\ ${ }^{a}$ Departamento de Ingeniería en Metalurgia y Materiales, Instituto Politécnico Nacional, Ciudad de México, México \\ ${ }^{\mathrm{b}}$ Dept. Polymeric Nanomaterials and Biomaterials, ICTP-CSIC, Madrid, Spain \\ ${ }^{\mathrm{c}}$ CIBER-BBN, Madrid, Spain
}

\section{A R T I C L E I N F O}

\section{Keywords:}

Alginate hydrogel

Injectable

Composite

Bone tissue

Hybrid

Bioprinting

\begin{abstract}
A B S T R A C T
This review focuses on recently developed alginate injectable hydrogels and alginate composites for applications in bone tissue regeneration, and it evaluates the alternatives to overcome the problems that avoid their utilization in the field. Section 2 covers the properties of alginates that have made them useful for medical applications, in particular their ionic gelling ability for preparing injectable compositions used as delivery drugs systems. The advantages and shortcomings of these preparations are revised together with the chemical modifications assayed. Section 3 describes how it has been taken advantage of alginates into the new field of biofabrication and the developments in bone engineering. The state of the art of this field is reviewed. Finally in Section 4, new developments and approaches that in opinion of the authors can lead to a breakthrough in bone tissue engineering using alginates are introduced.
\end{abstract}

\section{Introduction}

Bone is the most commonly transplanted tissue and, together with cartilage, are the two tissues with most attempts to be replicated, followed by the skin. However, no fully functional equivalents have yet been achieved that allow vascularization and integrate completely inside the body (Roseti et al., 2017). The complexity of the bone and cartilage tissues is thought to be directly related to the failing of the attempts made to fabricate artificial devices that replace them up to date (Henkel et al., 2013). Bone substitute materials (BSM) currently used include natural origin materials, synthetic (alloplastic) materials, composite materials and BSM containing living cells (Kolk et al., 2012). The requirements that BSM must include are: to have osteoinductive three-dimensional structure, to be able to contain osteogenic cells and osteoinductive factors, to provide sufficient mechanical properties and to promote vascularization (Kang et al., 2016). However, not all these requirements have been achieved simultaneously to date.

Among the materials applied for the efficient performance of scaffolds for tissue engineering including biological clues, the use of biomaterials with high water content like hydrogels represent a major proposal. Their soft nature, pliability and porous structure allow the effective diffusion of nutrients and oxygen into their structure, imitating the biological tissues (Buwalda, Dijkstra, Feijen, Vermonden, \&
Wim, 2014; Dragan, 2014). Additionally, hydrogels allow the introduction of other simultaneous strategies for tissue restoration through therapeutic delivery of proteins, growth factors and drugs over a prolonged period of time (Caló \& Khutoryanskiy, 2015; Yang, Junseok, Hwang, Hoffman, \& Hahn, 2014). Currently, much attention has been paid on injectable hydrogels that can be administered in a minimally invasive manner, increasing patient comfort and reducing recovery times, which leads to lower health care costs compared to subcutaneous implantation of preformed scaffolds (Küçüktürkmen, Öz, \& Bozkir, 2017; Radhakrishnan, Subramanian, Krishnan, \& Sethuraman, 2016; Vo et al., 2016).

In addition, due to their viscoelastic properties, injectable hydrogels are also attractive for Additive Manufacturing Technologies (AMT) Biofabrication (Bf) and Bioprinting (Groll et al., 2016). The "construction blocks" of three-dimensional scaffolds in bioprinting are called "Bioinks", and the most commonly used bioink materials are hydrogels. However, there are few systems on current clinical application or materials fitted for their application in bioprinting (Liu et al., 2017).

Alginate hydrogels are the most assayed materials for bone tissue engineering (BTE) and bioprinting due to their properties such as gelling capacity, low toxicity, high availability and low cost. They provide an appropriate niche for cell loading and, in addition, their inherent ionic crosslinking make them injectable which offers advantages over

\footnotetext{
* Corresponding author at: Departamento de Ingeniería en Metalurgia y Materiales, Instituto Politécnico Nacional, Ciudad de México, México.

E-mail addresses: acitlalli89@gmail.com (A.C. Hernández-González), ltellezj@ipn.mx (L. Téllez-Jurado), luis.rodriguez-lorenzo@ictp.csic.es (L.M. Rodríguez-Lorenzo).
} 
other materials used as solid scaffolds. Alginate polysaccharide has been declared safe, by the United States Food and Drug Administration (FDA) (Xu \& Lam, 2018), for application in humans (de Vos, Lazarjani, Poncelet, \& Faas, 2014). However, no alginate devices for BTE have been developed so far. Bioprinting using alginates as a bioink is a new opportunity for these hydrogels to expand their applications in bone regeneration.

This paper revises recently developed alginate injectable hydrogels and alginate composites for applications in bone tissue regeneration. It comprises ionic crosslinking, chemical modifications in the backbone and covalent crosslinking, the assay of composites and the preparation of bioinks. Finally, new potential directions that can be interesting for the field of Bioprinting with alginates are explored.

\section{Alginate hydrogels}

Alginate is a linear polysaccharide composed of homopolymeric units of 1,4-linked (-D-mannuronic acid) (M) and (-L-guluronic acid) (G) (Pina, Oliveira, \& Reis, 2015). M block segments posses a linear and flexible conformation; whereas the $(1 \rightarrow 4)$ linkages to guluronic acid introduce a steric hindrance around the carboxyl groups. For this reason, the $\mathrm{G}$ block segments provide folded and rigid structural conformation that is responsible for a pronounced stiffness of the molecular chains. High $\mathrm{M}$ content alginates are also immunogenic and more potent in inducing cytokine production as compared to high $\mathrm{G}$ content alginates (Otterlei et al., 1991). Alginates extracted from different sources have different M and G contents along with the length of each block, influencing the properties of the material (Pina et al., 2015). For example, while algal alginates usually show a high content of $\mathrm{G}$ blocks, alginate produced by pseudomonas aeruginosa does not possess $G$ blocks. A significant structural modification of alginates is the natural acetylation in the $0-2$ and/or O-3 positions, which have been so far reported only in bacterial alginates (Fig. 1), i.e. acetylating algal alginates via chemical treatments. The intrinsic viscoelasticity of alginates depends on the frequency of the constituting blocks. Flexibility decreases as follows: MG block > MM block > GG block. An extensive explanation can be found in supplementary material 1 and references (Stokke, Smidsrød, \& Brant, 1993; Smidsrød, Glover, \& Whittington, 1973; Vold, Kristiansen, \& Christensen, 2006; Whittington, 1971)

They are alginates with a polymerization degree in the range 50-3000, corresponding to molecular weights of approximately 10-600 kDa (Imeson, 2010; Lee \& Mooney, 2012). Alginate aqueous solutions have non-Newtonian characteristics (Becker \& Kipke, 2002), with higher viscosity as the $\mathrm{pH}$ decreases, obtaining maximum values at $\mathrm{pH}$ of 3.0-3.5. Alginates are likely to show $\mathrm{pH}$ responsive properties due to the presence of carboxyl groups on its backbone. The $\mathrm{pH}$ sensitive behavior is evident because higher swelling ratios are observed at higher $\mathrm{pH}$ values. The higher swelling is attributed to ionic carboxylate groups in the backbone, mainly due to the protonation of carboxylic acids in the polysaccharide (Sun \& Tan, 2013). Outstanding properties of alginate materials include their ability of undergoing in situ gelation (Axpe \& Oyen, 2016), water solubility (Rhim, 2004), cytocompatibility (Gonzalez-Pujana, Orive, Pedraz, Santos-Vizcaino, \& Hernandez, 2018; Klöck et al., 1997; Lee \& Mooney, 2012), mucoadhesive nature (Gonzalez-Pujana et al., 2018), prolonged release of active agents (Gombotz \& Wee, 1998; Osmokrovic et al., 2018; Stockwell, Davis, \& Walker, 1986) and protective barrier for cell and particle release systems (Leijs et al., 2016; Qi et al., 2012; Qiu et al., 2017). Thus, alginate hydrogels can be used for a wide range of applications such as drug carriers (Guan et al., 2018; Kurczewska et al., 2017), wound dressing (Rezvanian, Amin, \& Ng, 2016), matrices for periodontal application (Gruskin, Doll, Futrell, Schmitz, \& Hollinger, 2012), arthroscopic applications (Su, Liu, \& Yeh, 2017), etc. In addition, alginate hydrogels are studied as minimally invasive treatments (Lee, Lee et al., 2018; Park, Kang, Kim, Mooney, \& Lee, 2009) for bone tissue regeneration. However, the main disadvantage is their difficult degradation, related to the use of high molecular weight alginates in order to obtain mechanical properties similar to those of hard tissues.(Szekalska, Puciłowska, Szymańska, Ciosek, \& Winnicka, 2016). Considering that the alginate materials have the inconvenient of showing slow and incomplete degradation and that molecular weight of the majority of commercial alginates is above the renal clearance threshold (> $50 \mathrm{kDa}$ ), (Bouhadir, Kruger, Lee, \& Mooney, 2000; Gao, Liu, Chen, \& Zhang, 2009), multiple efforts have been made over the last decade in order to find a compromise between the mechanical properties and the degradation kinetics (and potential toxicity of degradation products) of alginate hydrogels by varying crosslinking methods, alginate molecular weight, chemical structure and processing technologies. Next, we will describe an overview of alginate hydrogels preparation and the most frequent attempts to overcome alginate hydrogels disadvantages.

\subsection{Alginate hydrogel synthesis by ionic crosslinking}

Alginate hydrogels are commonly prepared by ionic crosslinking through combination of alginate with divalent cations. These, cooperatively interact with $\mathrm{G}$ monomers blocks to form ionic bridges. In a solution of alginate, blocks of $\mathrm{M}$ monomers form weak junctions with divalent cations. However, the interactions between G monomers blocks and divalent cations form tightly held junctions. The mechanism consists in the coordination of divalent ions with four-carboxyl groups to form an egg-box arrangement (Grant, Morris, Rees, Smith, \& Thom, 1973). There are two possibilities of obtaining alginate hydrogels through ionic gelation: internal and external gelation (Fig. 2).

Internal gelation is preferred to promote in situ forming hydrogels and it is suitable for injectable alginate applications. In this mechanism, a controlled gelation is carried out by the use of divalent cationic salts

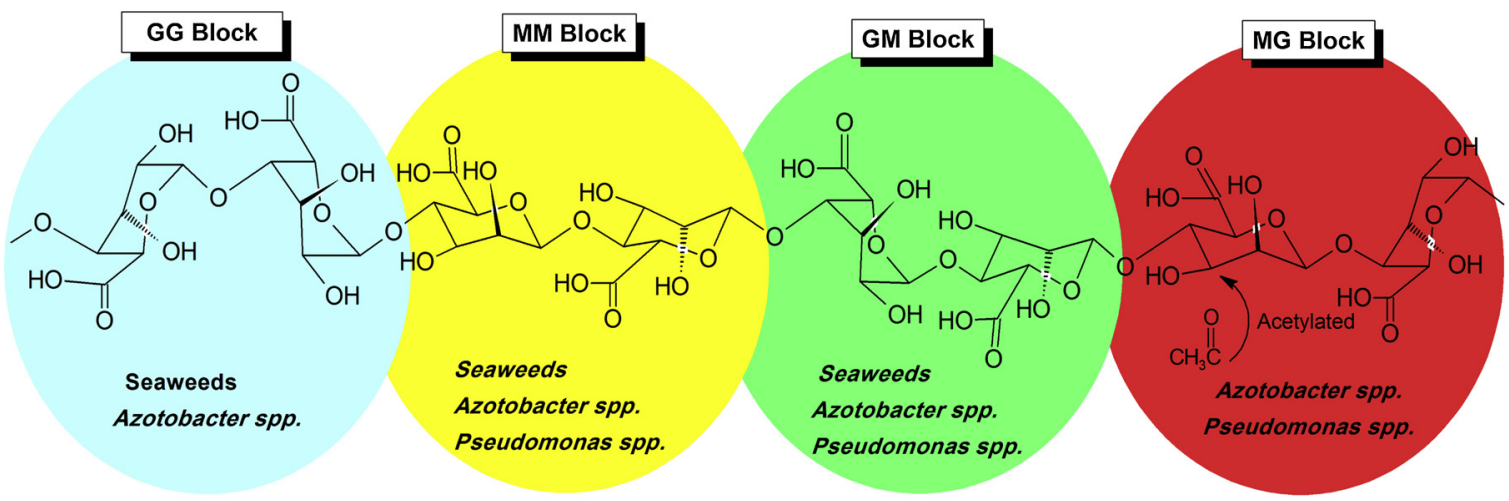

Flexibility

Fig. 1. Alginate chemical structures with different conformational blocks and their natural extraction sources. 
(a)

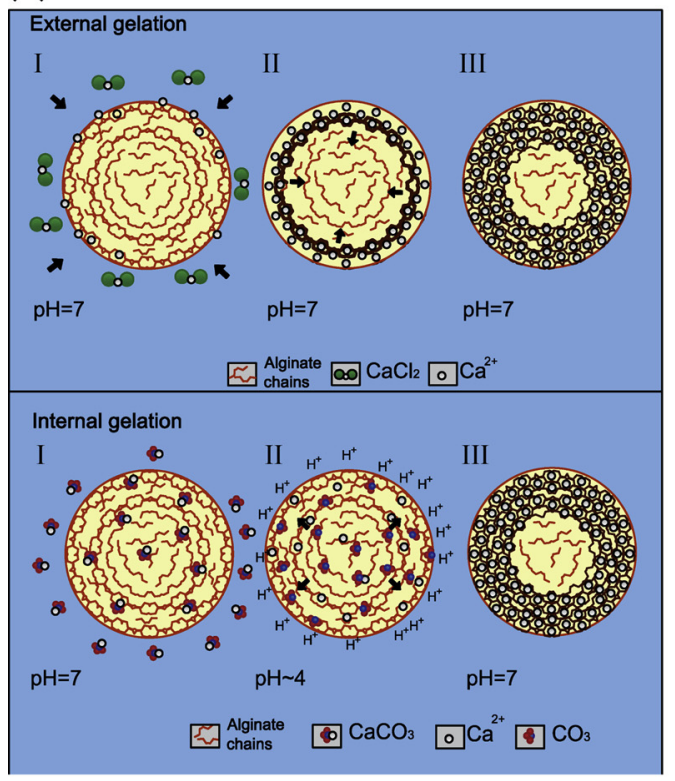

(b)

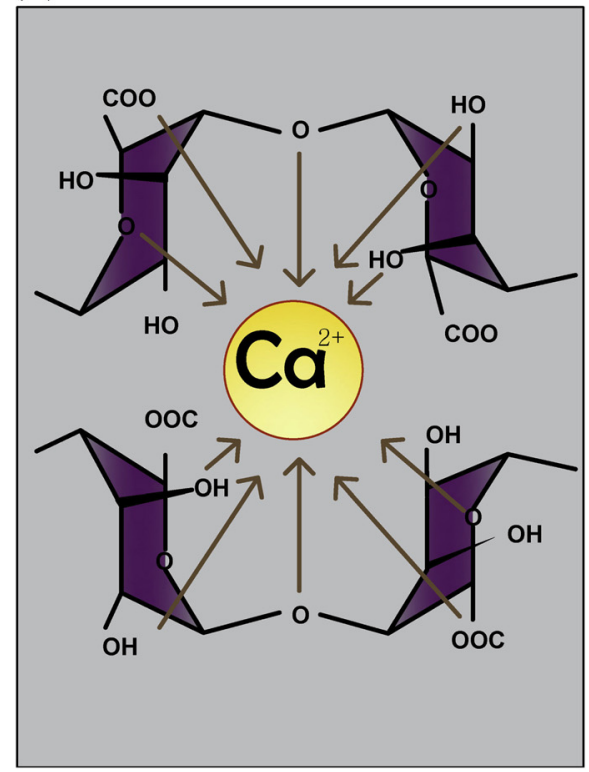

Fig. 2. Ionic gelation of alginate polysaccharides a) Gelling mechanisms and b) Egg-box model.

of low solubility at neutral $\mathrm{pH}$, with a subsequent acidification of the medium to release the cations allowing a better control of gelation kinetics and resulting in hmogeneous gels

In a paradigmatic study, Kuo and Ma (2001) synthesized alginate gels through the internal gelation in order to study the crosslinking density and compression properties of the gels as a function of alginate source, alginate concentration, calcium content and temperature. They used sodium alginate of high guluronate content (LH), or sodium alginate of high molecular weight (MP). The process consisted in the addition of glucono- \& lactone (GDL) which slightly decreased the $\mathrm{pH}$ of the solution, inducing the release of calcium cations previously added for their uniform distribution before gelation. Obtained results showed that the crosslinking density is higher at lower gelation rates, obtaining stable gels with lower concentration of both calcium ions and alginate. Also, it was noticed that compressive modulus increases as alginate concentration does (from 25 to $185 \mathrm{kPa}$ in the LH alginate range from 1.5 to $4.5 \mathrm{wt} / \mathrm{v} \%)$. Finally, it was reported that at the same polymer concentration (1.5 $\mathrm{wt} \%$ ), depending on the calcium/COOH molar ratio, both the molecular weight and the guluronate content influence the mechanical properties of the hydrogels. At low calcium contents, the $M P$ alginate gels showed higher compressive modulus than $\mathrm{L} H$ alginate gels ( $\sim 14$ and $4 \mathrm{kPa}$, respectively), while at calcium contents above of 0.27 calcium/COOH molar ratio, the compressive modulus of $M P$ alginate gels is lower than those of $\mathrm{LH}$ alginates (35 and $85 \mathrm{kPa}$, respectively). On the other hand, as an alternative to GDL, these authors propose the use of water-soluble calcium chelating agents. These agents, after exposure to ultraviolet (UV) light, undergo an irreversible molecular change that decreases their affinity for calcium $\left(\mathrm{Ca}^{2+}\right)$ allowing the cation release for crosslinking (Bidarra, Barrias, \& Granja, 2014). For instance, $\mathrm{Ca}^{2+}$ is extensively used to prepare ionic crosslinked alginate hydrogels (Bidarra et al., 2011; Fonseca, Bidarra, Oliveira, Granja, \& Barrias, 2011; Oliveira et al., 2008), but the effect of the ionic crosslinker on the properties of the hydrogel is still a matter of study. Chan, Jin, and Heng (2002) studied the combination of alginate microspheres with calcium chloride and zinc sulfate salts for drug release applications. The authors observed that $\mathrm{Zn}$ cations interact with alginate at different sites with respect to Ca cations, being less selective but allowing more extensive crosslinking.

Straccia, d'Ayala, Romano, and Laurienzo (2015) developed sodium alginate based hydrogels crosslinked with carbonate and zinc hydroxide in presence of GDL, for wound coating application. The obtained hydrogels present higher water retention compared to those of crosslinked with free Ca ions. However, lower values of ultimate stress are observed (from 4500 to 22,500 Pa), as well as low stability in Phosphate Buffer Solution (PBS), requiring an additional chemical treatment with calcium chloride to improve the in vitro stability. The authors explained that the calcium ions show a high specificity in the alginate crosslinking, leading to the formation of a tougher and rigid polymeric network than those synthesized with zinc cations.

On the other hand, the external gelation is the other ionic mechanism used to synthesize materials applied in bone tissue engineering, being widely applied for alginates processed in AMT (Section 2), but not commonly applied in the synthesis of injectable hydrogels for minimally invasive treatments. This type of ionic crosslinking has the advantage of being a simple low-cost process, due to the fast interaction between divalent ions and alginate. However, non-homogeneous hydrogels with low mechanical properties are frequently obtained, due to the generation of non-crosslinked alginate zones embedded into the crosslinked alginate layers. Currently, several researches are trying to overcome the disadvantages of this gelling mechanism. In 2016, Bajpai, Shukla, and Bajpai (2016) prepared alginate hydrogels by a diffusion technique through a dialysis tube, crosslinking with $\mathrm{Ca}^{2+}, \mathrm{Ba}^{2+}$ and a mixture of both ions. The obtained hydrogels were homogeneous with stability times in solution greater than $18 \mathrm{~h}$. It was observed that as the amount of $\mathrm{Ba}$ ions increases, the swelling ratio of the material decreases. Barium hydrogels showed greater structural stability in solution than calcium ones, explained as a consequence of the greater ionic radius of $\mathrm{Ba}$ ions, which are adapted in a better way in the cavities of the "box egg" structure of alginate gels. This work presents a possibility to fabricate porous scaffolds with specific geometries where the dialysis membrane may act as contention boundary that controls the gelling rate.

At the beginning of 2017, Rezvanain, Ahmad, Amin, and Ng (2017) synthesized alginate/pectin based hydrogel films for application as wound dressing. Hydrogels were ionically crosslinked with different concentrations of calcium chloride from 2 to $20 \mathrm{~min}$ and loaded with simvastatin. They showed that the hydrogel film with $0.5-1 \mathrm{wt} \%$ of calcium chloride and crosslinked in 2 min showed adequate mechanical properties with good physical integrity, a high water absorption capacity, no cytotoxicity and controlled release of simvastatin. Additionally, the crosslinking with calcium ions improves the tension stress of the films (from 2.87 to $9.28 \mathrm{~N} / \mathrm{mm}^{2}$ ) but decreases the elasticity, reducing 


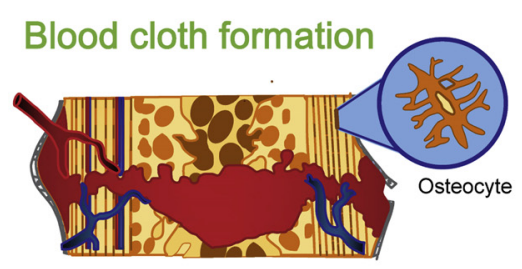

\section{Cleanning around fracture} and chemotaxis

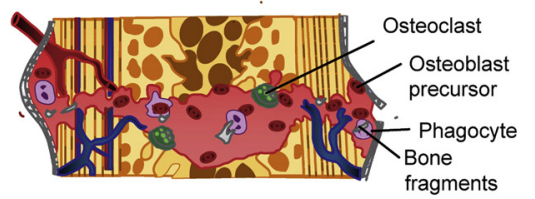

Provitional soft callus form

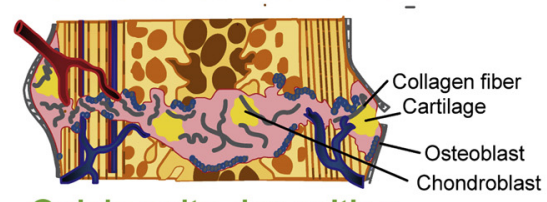

Calcic salts deposition

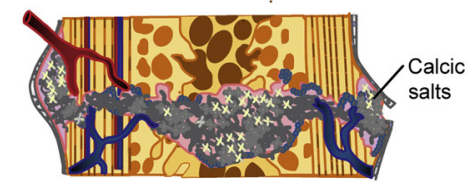

Hard callous formation

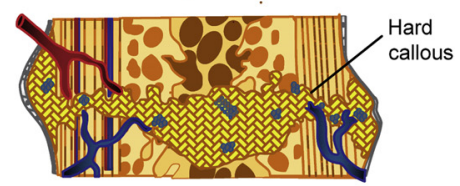

Bone remodelation

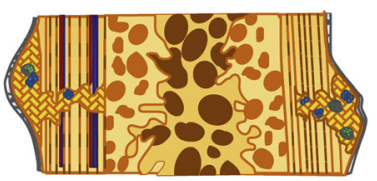

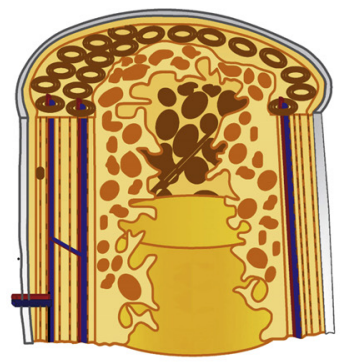

\begin{tabular}{|c|c|c|c|}
\hline Stage & $\begin{array}{l}\text { Source or } \\
\text { Trigger }\end{array}$ & $\begin{array}{l}\text { Protein, growth } \\
\text { factor, cytokine }\end{array}$ & Function \\
\hline & Osteocytes & Wnt signaling & Controls de production of OPG receptor \\
\hline & & $\begin{array}{l}\text { OPG decoy } \\
\text { receptor }\end{array}$ & $\begin{array}{c}\text { Reception of key osteoclast differentiation } \\
\text { factor RANKL }\end{array}$ \\
\hline & & $\begin{array}{l}\text { Wht anatgonist } \\
\text { SOST } \\
\text { DKK1 }\end{array}$ & $\begin{array}{l}\text { Regulate bone formation through } \\
\text { osteoclastogenesis inhibition }\end{array}$ \\
\hline \multirow{5}{*}{$1 \mid$} & Osteocytes & Wnt5a & Stimulate osteoclastic differentiation \\
\hline & \begin{tabular}{|c|}
-Osteoclast \\
-Response to \\
increased \\
rankl \\
\end{tabular} & $\begin{array}{l}\text { Sema4D- } \\
\text { Plexin B1 }\end{array}$ & Inhibits osteoblast differentiation \\
\hline & $\begin{array}{c}\text {-Response to } \\
\text { osteoclast } \\
\text { activity }\end{array}$ & $\begin{array}{l}\mathrm{Ca} \\
\mathrm{TGF}-\beta 1 \\
\mathrm{IGF}-1\end{array}$ & $\begin{array}{l}\text { Grouping of pluripotent mesenchymal } \\
\text { cells (pre-ostoblast) }\end{array}$ \\
\hline & Osteoclast & Wnt10b & $\begin{array}{l}\text { Pre-ostoblasts are induced to migrate } \\
\text { reception sites }\end{array}$ \\
\hline & Pre-osteoblast & OPG and RANKL & $\begin{array}{l}\text { Inhibits osteoclastogenesis and therefore } \\
\text { bone resorption }\end{array}$ \\
\hline \multirow{3}{*}{$\|$} & Pre-osteoblast & BMP-2, BMP-7 & Osteoblast differentiation \\
\hline & Osteoblast & BMP's & Expression and secretion of VEGF- $\alpha$ \\
\hline & & $\begin{array}{l}\text { TGF, DGF, } \\
\text { FGF-1, IGF }\end{array}$ & $\begin{array}{c}\text { Induce fibroblast proliferation and } \\
\text { chondrocyte differentiation (Soft callous) }\end{array}$ \\
\hline \multirow{2}{*}{ IV } & Osteoblast & BMP-5 to BMP-7 & Boost BMP-2 secretion \\
\hline & & BMP-2 & $\begin{array}{l}\text { Induce osteoblast differentiation to ostoid } \\
\text { matrix }\end{array}$ \\
\hline & Osteoblast & $\begin{array}{c}\text { OPG } \\
\text { RANK-RANKL }\end{array}$ & $\begin{array}{l}\text { Regulate hard callous remodelation } \\
\text { through stimulation and inhinition of } \\
\text { osteoclastogenesis }\end{array}$ \\
\hline
\end{tabular}

Wnt - Wingless-related integration site;OPG - Osteoprotegerin; SOST - Sclerostin; DKK1 - Dickkopf-related protein 1; RANKL - Receptor activator of nuclear factor kappa-B ligand; TGF- $\beta 1$ - Transforming growth factor- $\beta 1$; IGF-1 - Insulin-like growth factor-1; 1,25-Vit D3 - 1,25-dihydroxyvitamin D3; RANK - Receptor activator of nuclear factor kappa-B; BMP - Bone morphogenetic protein; VEGF- $\alpha$ - Vascular endothelial growth factors

Fig. 3. Healing and consolidation process of damaged bone tissues.

deformation values from 55.8 to $19.6 \%$ at fracture stress.

Ionically crosslinked alginate hydrogels do not have the required mechanical strength to be applied in bone engineering as it will be explained later. Nevertheless, they have similar mechanical properties to ECM, which explains why they have emerged as a platform for studying cell response in tissue engineering testing, including bone engineering.

2.1.1. Alginate hydrogels as delivery systems of bioactive molecules and cells for bone regeneration

When a bone fracture occurs, the human body starts a consolidation process in order to regenerate the damaged tissue. The successful bone regeneration must combine viable cells, extracellular matrix, specialized cytokines and growth factors that induce adequate mechanical and vascular environments (Fig. 3). The lack of one or more of these elements involves the failure of the consolidation process. Some conditions associated to the failure in bone tissue regeneration are: lack of space, obstruction in cell and growth factors delivery caused by the collapse of surrounding tissues, deficiency of calcareous salts, pseudo arthrosis, inhibition of growth factors or injury with massive bone loss and carcinogenic nature in the bone (Silberman \& Varaona, 2011; Welsch \& Sobotta, 2008). An approach to overcome these difficulties consists on encapsulating specific bioactive cells and molecules in alginate gels for transport and direct application in the damaged area. In this way, an efficient and rapid recovery is sought to reduce complications associated with the instability of the clot due to micro-movements during the healing phase.

It is important to consider that bone regeneration is dependent on the cell line used and its interaction with the material. The first studies to report bone cell culture in a 3-D alginate matrix were carried out by 
Majmudar, Bole, Goldstein, and Bonadio (1991), using hydrogels crosslinked with calcium chloride and Mouse Calvarial 3T3 osteoblastic cell line (MC3T3). In this study, the cellular viability for as long as 8 months and the mineralization of an extracellular matrix in vitro that contained fibronectin, type III collagen, and type I collagen were observed. Later in $2001 \mathrm{Kuo}$ and Ma (2001) developed in situ gelation alginate materials with efficient encapsulation of MC3T3 cells and adequate control in the gelation kinetics. Markusen et al. (2006) demonstrated that isolated cells from bone marrow could be entrapped within alginate/glycine-arginine-glycine-aspartic acid-tyrosine (GRGDY) beads, and it shows cell viability, in different culture vessels type, above $80 \%$ at 15 days of culture. However, no cell proliferation for alginate or modified alginate hydrogels was observed. Some other studies explored the osteogenesis influenced by different cell lines including cells derived from adipose tissue (Awad, Wickham, Leddy, Gimble, \& Guilak, 2004) added with platelet-rich plasma (Man et al., 2012), periodontal and human gingival stem cells (Moshaverinia et al., 2012), co-cultures of endothelial cells and bone cells (Grellier et al., 2009), etc.

Growth factors significantly conduct the stem cell differentiation, so their addition to the damaged site could enhance the body healing processes. Based on this, several investigations propose the growth factors delivery encapsulated in alginates into the damaged area. Such is the case of the research developed by Ansari et al. (2017) who developed a stem cell delivery system based on alginate/hyaluronic acid loaded with Transforming Growth Factor Beta (TGF- $\beta 1$ ), encapsulating Periodontal Ligament Stem Cells (PDLSCs) for chondrogenic differentiation. The results showed a continue release of TGF- $\beta 1$, chondrogenic differentiation up to 14 days, and higher expression level of chondrogenesis-related genes for hydrogels with $2: 1 \mathrm{w} / \mathrm{w}$ ratio alginate/HA. The authors analyzed the role of the microenvironment and the presence of inductive signals for viability and differentiation of Mesenchymal Stem Cells (MSCs), demonstrating that the hydrogel elasticity can contribute to the chondrogenic differentiation of MSCs.

Segredo-Morales et al. (2018) prepared injectable thermo-responsive hydrogels for regeneration of osteoporotic bone defects. The hydrogel were composed by ionically crosslinked alginate/poloxamine, containing microspheres of poly(lactide-co-glycolide)/poly(lactide) loaded with $17 \beta$-estradiol $(\beta E)$ and/or Bone Morphogenetic Protein (BMP)-2. AEach of the systems present drug release during 6 weeks divided in one quick release (three days) followed by a long slow phase. In vivo evaluation in rats with osteoporotic and non-osteoporotic bone defects, showed a synergic effect between $\beta E$ and BMP-2, yielding a higher percent of drug/growth factor release for the formulations containing both $\beta E$ and BMP-2.

On the other hand, Gothard et al. (2015) assayed a demineralizeddecellularized bone supplemented with alginate and bone marrow stem cells enriched with stro- 1 and growth factors encapsulated in poly (lactide-co-glycolide) (PLGA) microparticles (VEGF, TGF- $\beta 3$, BMP-2, PTHrP and VitD3). No significantly promotion of bone formation from the addition of exogenous growth factors compared to alginate hydrogels containing only extracellular bone matrix was observed. The most efficient materials were those supplemented with BMP-2 and VitD3.

Attacking specific pathologies and reducing the risk of infection, antimicrobial agents and/or particles resistant to infections have been encapsulated as well in alginates. An early work in this context, was presented by Zhang et al. (2008). Scaffolds coated with vancomycin loaded ionically crosslinked alginate hydrogels were synthesized. The scaffolds showed a sustained drug release profile depending on the alginate concentration. Additionally, a standardized bacterial assay showed osteoblast proliferation and drug activity after encapsulation.

In 2013, Morais et al. (2013) developed injectable hydrogels composed of alginate, alginate/chitosan and alginate/hyaluronate incorporating Ce III ions. The alginate /hyaluronate hydrogels showed greater proliferation of the cell line Homo sapiens bone osteosarcoma MG63. In addition, the Ce (III) ions induced an antimicrobial activity when they were evaluated against the streptococci Staphylococcus aureus, Staphylococcus epidermidis, Pseudomonas aeruginosa and Candida albicans.

In 2016, Rescignano et al. (2016) developed alginate hydrogels crosslinked with $\mathrm{CaCO}_{3}$ and GDL, encapsulating silver nanoparticles at different concentrations. The effect of silver particles in the antimicrobial activity and rheological properties of the hydrogel was evaluated. The hydrogels showed to be effective against the bacterium Escherichia coli and Pseudomonas aeruginosa with inhibitory zones between 5 and $7 \mathrm{~cm}$ after $24 \mathrm{~h}$ of incubation. On the other hand, it was determined that the materials with $2.5 \mathrm{wt} \%$ of silver nanoparticles increase the elastic modulus of the alginate hydrogels from 293 to $530 \mathrm{~Pa}$, indicating an interaction between the alginate network and the silver particles.

In 2017, Yan et al. (2017) obtained alginate/bacterial cellulose nanocrystal (BCN's) scaffolds by internal gelation in order to achieve a porous microstructure as well as the desired mechanical and biological activity of the composite scaffold. Biodegradative behavior and cell attachment were regulated by successive layer-by-layer electrostatic assembly of chitosan and gelatin on the surface of the scaffold.. The incorporation of BCN's improved the compressive strength, biodegradation rates, and increases the porosity from 71.2 to $77.4 \%$. In addition, the outer gelatin chains containing repetitive motifs of arginineglycine-aspartic sequences favored the attachment, proliferation and differentiation of osteoblastic MC3T3-E1 cells.

Based on all the former discussion, it can be stated that alginate hydrogels stand out due to their ability to encapsulate cells and bioactive molecules, allowing their use in combinational strategies.

\subsection{Chemically modified injectable alginate hydrogels}

Alginate hydrogels chemical composition and structure have been altered in order to tailor specific properties for their applications in tissue engineering. In order to improve degradability, mechanical properties, cell adhesion, and features for specific applications such as printability or drug release. Alteration and variation of the molecular weight, backbone chemical modifications or covalent crosslinking of alginates have been studied.

\subsubsection{Alteration and variation of molecular weight}

Alginate chemical composition is a key feature in relation to the final hydrogel properties, such as crosslinking density, toxicity or degradability; and the variation of the polymer $\mathrm{Mw}$ or the blending of oligomers are exploitable routes to control the final properties of the hydrogel.

One of the most representative studies of the molecular weight influence on the hydrogel properties was developed by Kong, Alsberg, Kaigler, Lee, and Mooney (2004). They analyzed the control over the degradation rate of ionically crosslinked alginate hydrogels by the combination of alginates of high molecular weight with low and high guluronate content (HMw-M and HMw-G) and a low molecular weight alginate (LMw-G) obtained from $\gamma$-rays irradiation. The authors reported that the degradation rate of hydrogels largely depended on the molecular weight of the guluronate blocks, but depended loosely on the total Mw. Binary hydrogels that have a mismatch in the size of crosslinking junctions exhibited faster decrease in the elastic modulus than hydrogels formed by segments of similar size. However, a decrease in the Mw of polymers while maintaining the difference in Mw of guluronate, increases the degradation rate of the gels. This suggested that the gels degradation kinetics can be modulated in a broad range with the proper selection of the G block size and the polymer molecular weight.

Bonino et al. (2011) reported the modification of high molecular weight alginate $(\mathrm{HMw}-196 \mathrm{kDa}, \mathrm{Mw} / \mathrm{Mn}=1.6)$ by gamma ray irradiation to obtain low molecular weight alginate (LMw-37 kDa, Mw/ $\mathrm{Mn}=1.5$ ). They fabricated nanofibers of different molecular weight by electrospinning for a possible application in bone tissue. It was 
observed that the low Mw polymer cannot be used for fiber preparation by electrospinning due to limited entanglements of this. The use of surfactants in high concentrations (up to $8 \mathrm{wt} / \mathrm{v} \%$ ) and highly hydrophobic was necessary for the manufacture of fibers. On the other hand, nanofibers prepared with both $\mathrm{HMw}$ and $\mathrm{LMw}$ and crosslinked with $\mathrm{CaCl}_{2}$ showed good processability and higher degradation rate. In 2017, $\mathrm{Xu}$ et al. (2017) studied the influence of the alginate Mw on its hemocompatibility for Human umbilical vein endothelial cells (HUVECs) viability. In this research, sodium alginates solutions were degraded using a heterogeneous phase acid process at different times, obtaining samples of different molecular weights with homogeneous molecular weight distribution. Alginates with intermediate molecular weight $(20,680-13,170 \mathrm{kDa})$ showed higher cell viability than $\mathrm{HMw}$ $(50,075 \mathrm{kDa})$ and $\mathrm{LMw}(1170 \mathrm{kDa})$. However, HMw alginates trend to solidify and induced thrombosis.

In this way, it has been determined that the molecular weight in alginates is an important parameter in the final properties of the material, being remarkable that alginates of higher molecular weight produce stiffer gels, and those of medium and low molecular weight allow a greater degradability and cell proliferation.

There are different routes to adjust MWs from alginate and derivatives, like enzymatic preparation (Falkeborg et al., 2014), ultrasonic irradiation (Feng, Cao, Xu, Wang, \& Zhang, 2017), ultraviolet photolysis [(Burana-osot et al., 2009)], oxidative-reductive depolymerization [(Li et al., 2010)], thermal degradation [(Kelishomi et al., 2016)] and breakdown using supercritical water [(Meillisa, Woo, \& Chun, 2015) 5], (Xu, Bi, \& Wan, 2016). However, it is important to mention that, although chain scission of alginates to lower molecular weight compounds increase their chances of being completely attenuated by the body, the alginates themselves possess the characteristic of not being naturally degraded by enzymes in mammals. Thus, it may take months before they are totally degraded at the implantation sites (Gao et al., 2009). For this reason, the introduction of labile groups within the alginate chain through chemical modification has been attempted.

An example of $\mathrm{Mw}$ reduction is the partial oxidation of sodium alginate to form oxidized sodium alginate (OSA). Ding, Zhou, Zeng, Wang, and Shi (2017) investigated the structure and properties of OSA samples with the intention of crosslinking alginate with collagen-fiber for biomedical applications. In this work, alginate was oxidized and later fractionated by graded ethanol precipitation to obtain four OSA factions with a narrow range of molecular weights. The molecular weight of the fractions decreased with increasing the ethanol concentration and the fractions with lower molecular weight had higher aldehyde contents. Sodium alginate oxidation and other chemical modifications for alginate hydrogels properties tuning are discussed in depth in the next section.

\subsubsection{Chemical modifications of the backbone}

Alginate chemical modifications have been developed with the purpose of granting some specific functionality for alginate based materials (Fig. 4). There are two different routes for alginates functionalization, through carboxyl and hydroxyl groups. In the first one, alcohols are esterified at high temperature in the presence of catalyst. This is a feasible mechanism to incorporate labile groups to the alginate structure (Yang, Xie, \& He, 2011). However, esterification is not deeply used in bone tissue engineering since the ester bond is prone to hydrolysis at high rates (Bu, Kjøniksen, Knudsen, \& Nyström, 2004). Propylene glycol alginate (PGA) is the only esterified alginate derivative commercially exploited. It is applied as stabilizer, emulsifier or thickener in food products and as an in situ gelling agent for drug delivery (Hadef, Omri, Edwards-Lévy, \& Bliard, 2017). However, since ionic heterojunction of esterified alginates are developed by the interaction of residual unmodified carboxyl groups, when PGA are obtained with high degrees of esterification, they exhibit poor gelling ability and low mechanical properties (Liu, Li, Mao, \& Gao, 2018). Phosphorylated alginates were developed through the hydroxyl modification route. This reaction have been used to induce hydroxyapatite nucleation for bone tissue regeneration (Coleman et al., 2011). Moreover, alginate sulfation was developed to generate an analogue for heparin-binding proteins; however, due to the ionic nature of the compounds, the resultant materials had extremely quick degradation, tendency to form weak gels, steric hindrance for covalent bonding, and poor ability for ionic crosslinking (Yang et al., 2011).

Nowadays, the oxidized alginates (OAs) are gaining attention due to their highly reactive groups and faster degradation rates compared to unmodified alginates. Alginate oxidation is a relatively simple procedure with easy purification and non-toxic effects. The oxidation is carried out using sodium periodate, obtaining two aldehyde groups in each oxidized unit by rupture of carbon-carbon bonds in the alcohol groups, As a consequence, a lower molecular weight and the oxidized uronate residues make OA susceptible to alkali catalyzed elimination (Reakasame \& Boccaccini, 2017). However, OA show lower mechanical properties than the unmodified alginate hydrogels. Bouhadir et al. (2001), for example, performed the oxidation of sodium alginate at $4.9 \%$ for evaluation of degradability and in vivo response of the hydrogel. They found a molecular weight reduction of the OA from $390 \mathrm{kDa}$ to $255 \mathrm{kDa}$, and a decrease in the compressive modulus from $150 \pm 13 \mathrm{kPa}$ to $754 \pm 21 \mathrm{kPa}$ with respect to non-oxidized gels. The change in the compressive modulus during degradability test indicated an increased degradation rate, starting at the 6th day of incubation. The authors observed that the oxidized materials were still able to gel in the presence of calcium ions via ionic bonding. However, the interaction between the polymer chains and the divalent ions in ionically crosslinked OA hydrogels decreases due to the reduction in the number of GG blocks in OA chains. It was reported that the formation of ionic bonds requires an average of 20 adjacent guluronate groups. Moreover, Gomez, Rinaudo, and Villar (2007) determined that the oxidation of alginate tends to be selective towards the guluronate units, decreasing the ability to ionically gel at oxidation values higher than $10 \%$. In addition, the oxidation degree is limited by the formation of hemiacetal groups and their interference in the reaction (Painter \& Larsen, 1970).

OA hydrogels, due to the multiple aldehyde groups present in the backbone, are highly reactive to form covalent bonding through a nucleophile attack that modifies the final polymer properties. The oxidation degree of covalently bonded hydrogels strongly affects the network crosslinking degree, density, mechanical properties, degradation profile, and swelling behavior (Reakasame \& Boccaccini, 2017).

Other chemical modifications of alginates include the activation of carboxyl or hydroxyl groups using carbodiimide chemistry to form covalent bonding through a Michael addition (Jeon, Bouhadir, Mansour, \& Alsberg, 2009). Activating agents, such as EDC.HCl or 1,1'carbonyldiimidazole (CDI) form reactive intermediates and react with amine groups. However, it is important to consider that chemical crosslinking agents are a major obstacle to be use in tissue engineering as injectable in situ scaffolds due to their toxicity to cells (Chen, Xing et al., 2017).

Some functional materials obtained through chemical modifications and further covalent bonding will be described in the next section.

\subsubsection{Covalently crosslinked injectable hydrogels}

The covalent crosslinking of hydrogels generates strong and nonreversible chemical bonds. Furthermore, introducing covalent crosslinking is a promising implement to achieve controlled mechanical properties in alginate hydrogels (Lee et al., 2000; Zhao, Huebsch, Mooney, \& Suo, 2010). On the other hand, materials covalently bonded generally do not present the ability to be injected for in situ forming scaffolds, restricting this application. Nevertheless, there are some exceptions such as photocrosslinkable and viscoelastic hydrogels with shear thinning behavior.

Injectable photocrosslinkable alginate hydrogels are characterized by the addition of reactive groups such as methacrylates, through several reactions including; amide formation with carbodiimide 

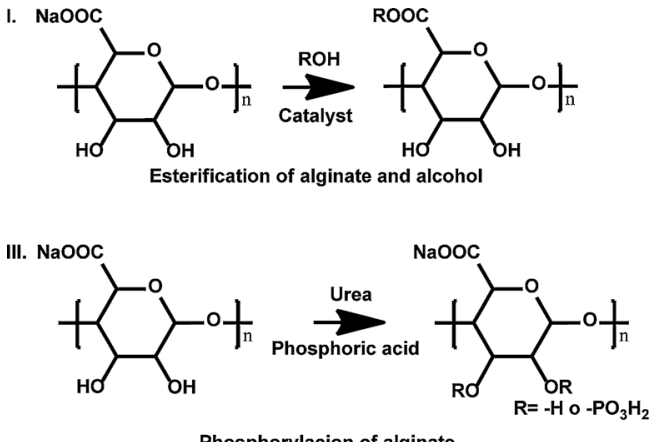

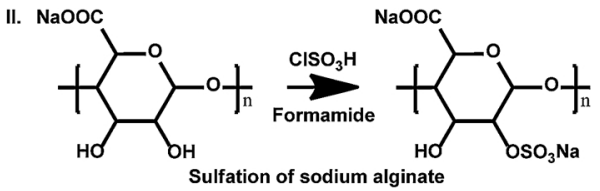

IV. ${ }^{\mathrm{NaOOC}}$

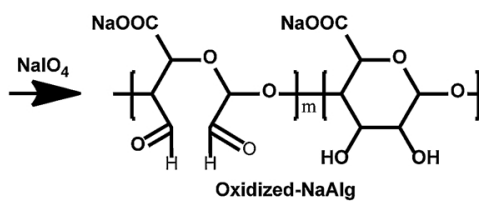

Oxidation of sodium alginate

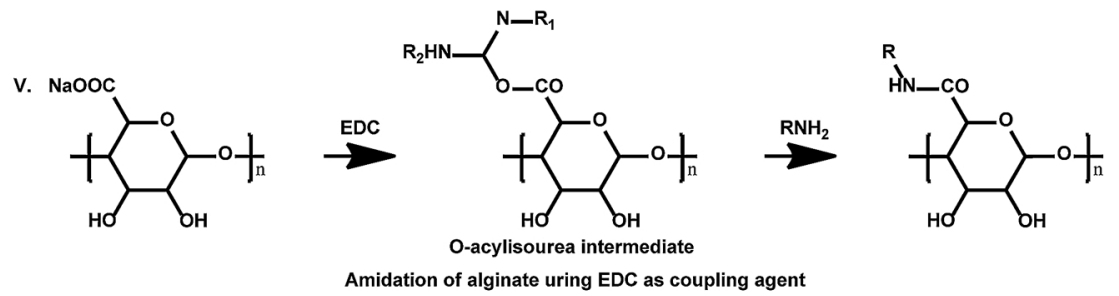

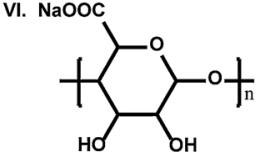

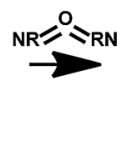

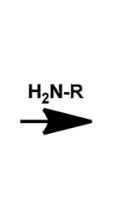

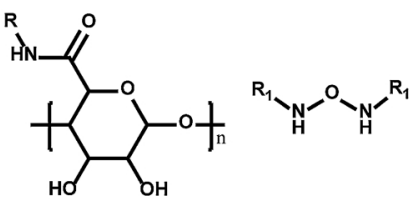

Carbodiamide mediated coupling of carboxylic acids to amines

Fig. 4. Chemical modification of alginate polysaccharide.

chemistry (Jeon et al., 2009), reductive amination, reaction of aldehyde groups in alginates after oxidation (Jeon, Alt, Ahmed, \& Alsberg, 2012), etc. In this method, a photosensitive system containing the reactive alginate prepolymer plus photoinitiators are applied by injection, and then subjected to a brief exposure of light, usually in the UV/Vis range to crosslink the material in situ. The process is explained as follows: the energy of the light excites the photoinitiators and triggers the formation of free radicals. Then, these reactive species propagate across the alginate prepolymer in solution, resulting in both, the generation of new free radicals and the establishment of crosslinks between the polymer chains. As the reaction proceeds, the number of crosslinks in the system increases and a network structure is obtained via a chain-growth mechanism (Pereira \& Bártolo, 2014). This procedure can be performed under mild conditions such as physiological $\mathrm{pH}$ and body temperature and also in contact with drugs or, under special conditions, cells. However, the principal drawbacks are the potential deleterious effects of light irradiation in cell viability (Lisby, Gniadecki, \& Wulf, 2005; Pfeifer, You, \& Besaratinia, 2005) potentiated by the requiring of good exposure to light after application to achieve efficient crosslinking. Cytotoxicity of radicals also generated by the dissociation of photoinitiators, local inflammation due to unreacted double bonds, lack of control over the crosslinking kinetic and resulting materials with oxygen inhibition (Lin \& Anseth, 2009).

Nowadays, researches are working to overcome these detriments through the selection of appropriate photoinitiators, light wavelengths, intensity and irradiation time. The purpose is to achieve a compromise between the crosslinking time and the cytocompatibility of the resultant materials (Pereira \& Bártolo, 2015). Desai, Koshy, Hilderbrand, Mooney, and Joshi (2015) modified the carboxylic acids of alginate backbone with tetrazine or norbornene to form covalently crosslinked alginate hydrogel networks using a bioorthogonal inverse electron demand Diels Alder click reaction. Additionally, thiol bearing peptides were added and photocrosslinking proceed via the norbornene modified groups and the photoinitiator Irgacure 2959. These materials produced a minimal inflammatory response and maintained their structural integrity over several months (Desai et al., 2015). Blue light initiators: camphorquinone (CQ), fluorescein (FR) and riboflavin (RF) have also been assayed as photoinitiators with low toxicity (Hu et al., 2012). Higher mechanical strength of CQ- or FR-initiated gels (compressive modulus 2.8 or $4.4 \mathrm{kPa}$, respectively), but a drastically reduced chondrocyte viability ( $5 \%$ and $25 \%$, respectively) were observed. Nevertheless, the use of radiofrequency crosslinking seems to reduce irradiation time from 120 to $40 \mathrm{~s}$, improving the compressive modulus without reducing cell viability.

The second line of research, the generation of shear thinning viscoelastic hydrogels include solutions of certain proteins, colloidal systems, peptides and polymer mixtures whose self-assembly behavior causes gelation in the material when it is not under shear stress (Radhakrishnan et al., 2016). Based on this property, the highly viscous polymer solution flows when a shear force is applied (injecting) and form a hydrogel when stops. The Alginates are an excellent base material for the generation of this type of materials due to their polyelectrolytic properties.

Some promising results also come from oxidized alginate covalently crosslinked with other free amino groups containing polymers (Wang, Wang, \& Teng, 2016; Wu et al., 2017; Yuan et al., 2017). Wei et al. (2015) developed self-healing hydrogels consisting of N-carboxyethyl chitosan-oxidized alginate-adipic acid dihydrazide linked by imine bonds obtained by Schiff base reaction between OA and CEC, as well as acylhydrazone bonds derived from reaction between $\mathrm{OA}$ and $\mathrm{ADH}$. These hydrogels are able of autorepair cracks with efficiencies up to $95 \%$. Hydrogels also demonstrate high viability of encapsulated $\mathrm{NIH}$ 3T3 fibroblasts, and great injectability, attributing the materials selfhealing nature to the coexistence of dynamic imine and acylhydrazone bonds in the hydrogel networks.

On the other hand, alginates do not have specific signal receptors 
for binding proteins so they exhibit poor cellular adhesion making it them prone to develop unwanted interactions, immunological response and proteolytic degradation (Tønnesen \& Karlsen, 2002). In order to solve this problem, the coupling of oxidized alginates with proteins such as collagen, fibronectin and gelatins have been proposed (Wright, De Bank, Luetchford, Acosta, \& Connon, 2014; Zhou \& Xu, 2011). Collagen-derived gelatins exhibit unique gelling properties due to the physical crosslinking of the triple helix structure of the native collagen. The gelatins retain informational signals and they are highly degradable in vivo, being possible to modulate their physicochemical properties. These characteristics, together with their high cellular adhesion, plasticity, availability and low cost have made gelatins one of the most popular companions for crosslinking with OA showing enhanced cytocompatibility in different kind of cells (Baniasadi, Mashayekhan, Fadaoddini, \& Haghirsharifzamini, 2016; Jia et al., 2014; Kim et al., 2012; Li et al., 2012; Sarker et al., 2014). However, even with gelatin, it is important to remember that the final properties of materials based on oxidized alginate are closely dependent on the oxidation degree of alginate. Balakrishnan and Jayakrishnan (2005) prepared injectable hydrogels based on OA and gelatin in presence of borax and analyzed the properties of the hydrogel with respect to the oxidation degree and compositions. They proposed that the presence of borax facilitates the base of Schiff's formation through hydroxyl complexation depending on the composition of the hydrogel. Additionally, the authors reported that the greater the oxidation degree, the faster is the gelling, obtaining hydrogels with high crosslinking degree and lower swelling. These materials showed $93 \%$ of metabolically active cells after $24 \mathrm{~h}$ on in vitro evaluation and an average pore size of $100 \mu \mathrm{m}$. As complement, in later works, Balakrishnan and Jayakrishnan (2005), Balakrishnan, Mohanty, Umashankar, and Jayakrishnan (2005), Balakrishnan, Joshi, Jayakrishnan, and Banerjee (2014) evaluated the hydrogels for application as wound healings and cartilage regeneration using hydrogel composition of $20 \%$ of oxidized alginate and $15 \mathrm{wt} \%$ of gelatin with alginate oxidation rate of $57 \%$. Hydrogels with a degree of swelling of $7.53 \pm 0.31$ and crosslinking density of $38.69 \pm 4.05$ were biologically tested observing mouse fibroblast cytocompatibility and rat hepatocytes proliferation. Sarker et al. (2014) synthesized hydrogel microcapsules of OA-gelatin using a phosphate-buffered saline solution (PBS) to maintain the $\mathrm{pH} \sim 7.4$, extending the gelation time of the hydrogel compared to those gels synthesized with borax. This strategy would allow to the OA/gelatin system to be applied in additive manufacturing technologies for scaffold fabrication.

Another approach in search of reducing associated disadvantages to the poor protein binding in alginates is their coupling with immobilized peptides with cellular recognition. The bioactive peptides will mimic both the adhesive properties of ECM proteins containing these sequences and the stimulation of cellular responses such as differentiation and proliferation. Some peptides have been identified for cell attachment. In particular, one of the first used and still most studied is the tripeptide sequence RGD (Arginine-Glycine-Aspartic acid) because of its abundance in adhesive proteins (e.g. fibronectin, laminin, fibrinogen, vitronectin), and its ability to bind to a wide variety of integrins. Alginate hydrogels can be functionalized with RGD through covalent crosslinking between the alginate carboxyl group and the peptide amino terminal groups (Rowley, Madlambayan, \& Mooney, 1999). The RGD peptide sequence increases the cell attachment and osteogenic differentiation in bone tissue regeneration as a function of the peptides density (Evangelista et al., 2007).

In 2015, Krishnan et al. (2015) performed a bone regeneration comparative study between a cortical autograft treatment and a perforated nanofiber mesh system with RGD-alginate and rhBMP. In this study and using a combinational approach (alginate-peptide-growth factor) it was determined that the alginate system has higher bone formation ability with respect to the autograft at 8 and 12 weeks. However, a lower local mineralization density as well as greater diffuse mineralization in the defect at all test times was observed. Besides, the group treated with NMA-rhBMP-2 presented higher torsional hardness and maximum torque compared with the autograft. The authors concluded that the treatment with alginate-RGD and rhBMP and the treatment with allograft have similar advantages.

In 2016, Dalheim et al. (2016) developed the coupling of bioactive peptide sequences (GRGDYP, GRGDSP and KHIFSDDSSE) to oxidized alginates $(8 \%)$ followed by reductive amination with pic-BH3 as an alternative to the traditional coupling mechanism based on carbodiimide chemistry, increasing the substitution degree from about $0.1-1 \%$ to $3.9-6.9 \%$ (mol peptide/mol uronic acid monomers) against the traditional coupling method. In this work, the authors showed the effect of high peptide densities from peptide-alginate hydrogels on the attachment of mouse skeletal myoblasts (C2C12) and human dental stem cells (RP89 cells). The authors reported that myoblasts were unaffected by the increase in peptide concentrations, but cluster on the hydrogel surface at the lowest peptide concentration were formed, and only the RP89 cells adhered on the alginate gels with the highest concentrations of peptide.

In summary, raw alginate hydrogels have drawbacks for their application in tissue engineering due to missing specific signal receptors for binding proteins, exhibiting poor cellular adhesion and difficult control of degradation kinetics. However, they can be tuned by chemical modifications incorporating appropriate "motivs" to the alginate hydrogel and/or covalent bonding.

On the other hand, mechanical properties similar to human bone is a requirement for bone tissue engineering. However, they have not been reached with alginate-polymer composites and barely explored in the injectable hydrogels field. Thus, some different approaches have been developed to correct this disadvantage, which are review below.

\subsection{Alginate -ceramic composites}

Materials for bone engineering should fulfill several requirements in order to be applicable. These requirements are: 3D structure to support cell infiltration and vascularization (Gross \& Rodríguez-Lorenzo, 2004), mechanical properties for the local specific tissue including the mechanical integrity maintained during the tissue healing and the appropriate degradation kinetics (Sánchez-Téllez et al., 2017). The surface should be bioactive, osteoinductive (Sánchez-Téllez, Tellez-Jurado, \& Rodríguez-Lorenzo, 2015), degradation products must be non-cytotoxic (Jiménez-Gallegos, Rodríguez-Lorenzo, Roman, \& Téllez-Jurado, 2017), and It should be able to contain osteogenic cells and deliver osteoinductive factors to build up a construct (Chen, Zhu, \& Thouas, 2012; Kang et al., 2016; Karageorgiou \& Kaplan, 2005). Most of the studies are directed to determine how the chemical composition and architecture influence cellular phenotype, differentiation, integration and extracellular matrix secretion during in vitro and in vivo assays (Lee, Langford, Rodriguez-Lorenzo, Thissen, \& Cameron, 2017). However, from the point of view of materials science, a single type of material can hardly provide all of these properties. In order to combine the advantages of different types of materials, the designing of composite materials have been investigated (Rodríguez-Lorenzo et al., 2009; Sánchez-Téllez, Tellez-Jurado, \& Chavez-Alcala, 2014). From a biomimetic perspective, the combination of organic and inorganic components is a natural strategy for manufacturing materials similar to bone tissue. Among the inorganic compounds used for the reinforcement of hydrogels, bioglasses (BG) and calcium phosphates are the most investigated.

Bioactive glasses are silica-based materials with a high surface reactivity, which enhances the capability to promote the nucleation and subsequent growth of calcium phosphate crystals, specifically bonelike apatite crystals, on their surface when exposed to similar physiological fluids (El-Rashidy, Roether, Harhaus, Kneser, \& Boccaccini, 2017; Sarker, Li, Zheng, Detsch, \& Boccaccini, 2016). In 2016, Sarker et al. (2016) developed freeze-dried scaffolds from OA-gel hydrogel matrix reinforced with a BG45S5 glass in order to control the degradability and 
enhance the mechanical strength of the scaffolds. It was noticed that BG45S5 facilitates the gelation, enhances crosslinking degree of OAgels and promotes apatite formation. The compressive stress and compressive modulus of scaffolds with BG increase from $326 \pm 49 \mathrm{kPa}$ to $908 \pm 17 \mathrm{kPa}$ and from $65 \pm 13 \mathrm{kPa}$ to $417 \pm 33 \mathrm{kPa}$ compared to oxidized alginate hydrogel matrix alone. Also, a decrease in the porosity values of 40 and $30 \%$ was observed. Materials with $1 \mathrm{wt} \%$ of BG show promotion of bone marrow-derived stromal cells. In 2017, Bai et al. (2017) fabricated a triple crosslinked injectable hydrogel (non covalent, acylhidrazone bond and DA click covalent crosslinking) introducing double modified alginate and bioglass in hydrogels based on chondroitin sulfate-polyethylenglycol. The alginate was modified via adipic dihydrazide condensation and metaperyodate oxidation. This hydrogel was compared with different combination of crosslinkers and evaluated via water uptake, mechanical properties, degradation and bone repair test in vivo for cranial bone repair. The results showed a storage modulus up to $\sim 4000 \mathrm{~Pa}$ for triple crosslinked hydrogels and exceed $4500 \mathrm{~Pa}$ for triple crosslinked hydrogels enriched with BG. Besides, the authors elucidate the degradation mechanism of each crosslinking type in relation to the $\mathrm{pH}$ medium, showing a mass loss of $\sim 50 \%$ for neutral medium in 30 days and suitable bone regeneration effect for the in vivo assay.

The silica $\left(\mathrm{SiO}_{2}\right)$ is the component of bioactive glasses with the ability to form apatite on the surface when submerged in SBF (Jayakumar, Prabaharan, Kumar, Nair, \& Tamura, 2011; Li et al., 1992). Schloßmacher et al. (2013) developed alginate hydrogel systems containing silica and embedded with bone osteoblast related to the $\mathrm{SaOS}_{2}$ and osteoclast (Raw 264.7). The hydrogels with $\mathrm{SaOS}_{2}$ cell retained their capacity to synthesize crystals. Silica increases the gene expression encoding for osteoprotegerin in the hydrogel matrix with encapsulated $\mathrm{SaOS}_{2}$ cells. Also, the authors use a NanoTest Vantage system for calculating Martens hardness and elastic modulus, obtaining values of $0.742 \pm 0.027 \mathrm{GPa}$ and $22.826 \pm 0.579 \mathrm{GPa}$, respectively. In a recent work (Lewandowska-Łańcucka et al., 2017), photo-crosslinked hydrogels for bone regeneration using alginate and methacrylated functionalized gelatin and methacrylamide and loaded with submicron silica particles. The materials supported the mitochondrial activity of MEFs and MG-63 with successful mineralization induced by silica particles. In addition, it was observed that the storage modulus of mixed polymeric matrices gelatin/alginate, were higher (6880-9030 Pa) than alginate only -based materials (178-238 Pa). The addition of silica particles at higher concentrations influenced significantly the storage modulus.

Other ceramic materials highly considered in bone tissue engineering are calcium phosphate ceramics. One of them is carbonated hydroxyapatite $\left(\mathrm{CO}_{3}{ }^{2-} \mathrm{Hap}\right)$, which is present in bone tissue, teeth, and tendons, providing stability and hardness (Dorozhkin \& Epple, 2002). These materials are characterized for their biocontactive nature, osteoconductivity and biodegradation properties depending on their composition and textural parameters. Bioactivity can be explained by a number of factors, of which serum protein adsorption to the surface of particles is important (Fernández-Montes Moraleda, Román, \& Rodríguez-Lorenzo, 2013; Fernández-Montes Moraleda, San Román, \& Rodríguez-Lorenzo, 2016; Plaza et al., 2016). The dissolution of calcium phosphates releases calcium and phosphorus ions into the microenvironment of the cells, favoring the formation of new bone which is also supported by the rough surface of the particles (Fedorovich, Wijnberg, Dhert, \& Alblas, 2011).

Lin and Yeh (2004) prepared composite porous scaffold based on alginate with hydroxyapatite (HAp) through a phase separation method. The authors observed that the addition of HAp in the alginate matrix significantly improves the mechanical strength (compressive strength and elastic modulus) and enhances the osteosarcoma cell adhesion and proliferation on the scaffolds. These scaffolds have an average pore size of $150 \mu \mathrm{m}$ and over $82 \%$ of porosity. In 2013, Marsich et al. (2013) prepared alginate-HAp composite scaffolds by internal gelation followed by a freeze-drying procedure loaded with silver particles in order to induce antimicrobial properties. The obtained scaffolds have an average pore size of $341.5 \mathrm{~nm}$ and a porosity of $80 \%$ and they show strong bactericidal effect against both Gram + and Gram - bacterial strains, without cytotoxic effect on the cell. In 2015, Sarker, Amirian, Min, and Lee (2015) fabricated oxidized alginate-gelatin-biphasic calcium phosphate hydrogel scaffolds by freeze-drying. They were loaded with different content of spherical HAp particles. Materials without HAp present higher porosity and interconnected pore structure than materials loaded with Hap (pore size average $\approx 100-250$ $\mu \mathrm{m})$. On the other hand, materials with higher granules contents resulted in a higher compressive strength (compressive modulus in dry condition $2.45 \pm 0.19 \mathrm{MPa}$, and compressive modulus in wet condition $51 \pm 0.39 \mathrm{MPa}$ ). HAp loaded materials showed high cell viability, cell attachment, proliferation and differentiation behavior of rat bone marrow derived stem cells (BMSC). Remarkably, a higher number of live cells was observed in the interface between particles and polymer matrix. Also, earlier bone formation was observed for $25 \mathrm{wt} \%$ HAp loaded scaffolds in critical size defects of rabbit femoral condyle.

Other researches have explored the use of alpha-tricalcium phosphate $(\alpha-\mathrm{TCP})$, which reacts with water to form crystals of calciumdeficient hydroxyapatite (CDHA). Douglas et al. (2018) propose that the CDHA crystals formed by hydrolysis of $\alpha$-TCP particles may be able to interlock mechanically to a greater degree than preformed HAp particles, resulting in greater compressive strength and hardness. This is discussed to be beneficial for osteogenic differentiation of bone-forming cells. Perez and Kim (2013) developed a core shell design of fibrous scaffolds made of alginate with $\alpha$-tricalcium phosphate for in situ cytochrome $\mathrm{C}$ protein loading and controlled delivery. The core shell structured fiber was produced by injection of the alginate- $\alpha$-tricalcium phosphate and posterior crosslinking with $\mathrm{CaCl}_{2}$. The material, presented an initial drug delivery burst at lower crosslinking time and $\mathrm{CaCl}_{2}$ concentration. Also, the incorporation effect of $\alpha$-TCP $(10,50$, and $75 \mathrm{wt} \%$ ) into scaffolds on the mechanical properties by dynamic analysis was investigated. Scaffolds show more elastic than viscous behavior. Scaffolds with higher amounts of $\alpha$-TCP, yielded higher mechanical properties. As an example, the storage modulus shifted from $\sim 80 \mathrm{kPa}$ without $\alpha$-TCP to $800 \mathrm{kPa}$ with 75 wt $\% \alpha$-TCP p suggesting that $\alpha$-TCP phase promotes stiffening in the alginate matrix.

On the other hand, calcium phosphate bone cements are extensively used in orthopedics. These biomaterials can be directly injected in the bone defect, but they show some deficiencies that hindered their clinical applications. The mechanical strength is often insufficient to provide adequate mechanical support to the defect site, having poor washout resistance and handling properties (Ishikawa, Miyamoto, Kon, Nagayama, \& Asaoka, 1995). In 2017, Xu, Wang, Zhou, Huan, and Chang (2018) developed an injectable bone cement based on tricalcium silicate/sodium alginate (C3S/SA) composites by taking advantage of the interaction between $\mathrm{Ca}$ ions and SA molecules. An interpenetrating double network of calcium hydrate silicate (CSH) and alginate hydrogel was formed to enhance the washout resistance, formability, injectability and compressive strength of C3S. The Ca-Alginate hydrogel has the capability of holding together the cement particles, impacting composite cement. However, also a retarding effect in the C3S/SA hydration process for high amounts of Ca-Alginate hydrogel is observed, affecting the mechanical strength. The cement C3S/1.0 wt \% of SA showed the highest compressive strength with a gradual increase from $\sim 26$ to $54 \mathrm{MPa}$. Finally, the C3S/SA composite cements showed apatite formation in simulated body fluid (SBF) and cell proliferation promotion.

In 2017, Qiu et al. (2017) prepared composite cements of alginate hydrogel and Sr-containing mesoporous calcium silicate nanoparticles (mCSn). Viscous sodium alginate solutions mixed with mCSn particles resulted in a thick paste difficult to inject. However, the addition of GDL, decreased the viscosity, avoided mCSn-alginate composites sticking, and increase their injectability up to $90 \%$ while maintaining 
an integrated shape. These materials exhibited micropores $\sim 10 \mathrm{~m} \mu$ and higher mechanical properties than the GDL-free ones (compressive strength of wet samples $\sim 25 \mathrm{kPa}$, compressive strength of dry samples $\sim 6.5 \mathrm{MPa}$ and Young's modulus $\sim 90-130 \mathrm{MPa}$ ).The cement induced the fast formation of calcium-deficient carbonated apatite of low crystallinity. It had significant cytocompatibility, low cytotoxicity, and could support hBMSC proliferation and osteogenesis differentiation.

Lee, Park, Won, Shin, and Kim (2011) found that the addition of alginate increases the hardening rate of calcium phosphate cements, showing shorter setting times. Compressive strength increased with incubation time, obtaining the highest value at 3 days and maintaining it up to 7 days of incubation. Particularly, compressive strength significantly increased from $\sim 15 \mathrm{MPa}$ to $60-70 \mathrm{MPa}$ after 3-7 days of incubation at higher contents of alginate. Also, significant rat bone marrow derived stromal cell adhesion, proliferation and increased bone associated gene markers such as collagen type-1, osteopontin and bone sialoprotein were observed. Additionally, it was noticed that the main advantage of alginate addition in common Portland cements facilitated shaping into microspheres or fibers.

Thus, alginate composites have shown promising results for bone regeneration, in particular related with mechanical properties and cellular interactions (Fig. 5) in comparison with alginate hydrogels. However, it is still necessary to optimize their properties in order to enhance bone regeneration and provide adequate mechanical support. Moreover, their application in minimally invasive treatments is restricted by their rheological properties.

\section{Tissue engineering, additive manufacturing and bioprinting}

Tissue Engineering (TE) requires the development of scaffolds with a complex, patient specific external geometry in combination with a precise control over the internal architecture, all performed under cytocompatible conditions. Bioprinting is an emerging technology that pretends the direct manufacturing of constructs with simultaneous incorporation of biological and substrate materials.

Worldwide, different kind of Additive Manufacturing Technologies (AMT) have been applied for bioprinting (Derakhshanfar et al., 2018). However, the application of a specific AMT depends on the operating principle of the applied technology and the processability of the bioink. "Bioinks" are defined as the conjunction of substrate materials with biological components, and must met biomaterials requirements, together with additional parameters in order to ensure printing capacity. Hydrogels with injection ability are the most widely applied biomaterials for bioprinting (Ashammakhi et al., 2019).

Combination of bioprinting methods and appropriate bioinks is essential for successful fabrication of tissues. Therefore, a brief overview of the three classes of AMT suitable for hydrogel bioprinting is included in Table 1. It covers the advantages, the disadvantages, and the required bioink properties which very much depend on the operating principle of the technique. Whether the reader is interested in further reading about bioprinting technologies, some excellent reviews are referred (Adepu et al., 2017; Ashammakhi et al., 2019; Billiet, Schelfhout, Van Vlierberghe, \& Dubruel, 2012; Jang et al., 2018; Moroni et al., 2017; Ozbolat \& Hospodiuk, 2016).

As described before, injectable alginate hydrogels may be used as an appropriate platform for cell seeding. However, no alginate devices for hard tissues TE have been developed so far. Bioprinting using alginate as a bioink is a new opportunity for these hydrogels to expand their applications in bone regeneration.

\subsection{Bioprinting with injectable alginate bioinks: Printability conditions}

Ribeiro et al. (2017) defined printability as the possibility to extrude a hydrogel and dispense it in a pattern with a satisfactory degree of shape fidelity. The latter defined as how the printed structure is matching the original design. Printability is directed by the rheological properties of materials and must be adjusted to the specific fabrication process to generate constructs with high shape fidelity (Paxton et al., 2017). Injectable alginate bioinks are one of the most used and successful materials for bioprinting due to their shear thinning character, rapid crosslinking ability and feasibility of printing viable cells. They are particularly effective for bioprinting with nozzle systems due to the capacity of protecting encapsulated cells through the process (Axpe \& Oyen, 2016). However, there are some requirements that alginate bioinks must meet to be used in the fabrication of effective 3D printed constructs (Jovic, Kungwengwe, Mills, \& Whitaker, 2019). Table 2, offers a compilation of some representative alginate bioink formulations described in literature and summarizes the parameters studied for the printability assessment. Bioink evaluation tests, printing parameters and biological performance of the printed structures.

For instance, alginate bioinks must have sufficient viscoelasticity to achieve injectability during the printing process (Rheological properties) and good shape fidelity to maintain the overall shape of the fabricated scaffold, after printing (Chung et al., 2013). Since the viscosity of alginate bioinks depend on the alginate concentration, the alginate molecular weight and the cell density loading, printability can be promoted by controlling these parameters (Axpe \& Oyen, 2016; Freeman \& Kelly, 2017). A first approach is the control of alginate concentration. However, it can influence negatively long-term biological performance at high concentrations. Park et al. (2017) studied the influence of alginate concentration M/G 1.6 with high and low molecular weight (High Alg $3.5 \times 10^{5} \mathrm{~g} / \mathrm{mol}$ and Low Alg $1.43 \times 10^{5} \mathrm{~g} / \mathrm{mol}$ ) on printing fidelity and cell viability of a 3D printing nozzle based system (Fig. 6). A greater printing capacity, stability and fidelity was obtained for hydrogels High Alg /3 wt \% alginate, and a 1:2 ratio (Low Alg: High Alg). The metabolic activity and proliferation of the cells (a)
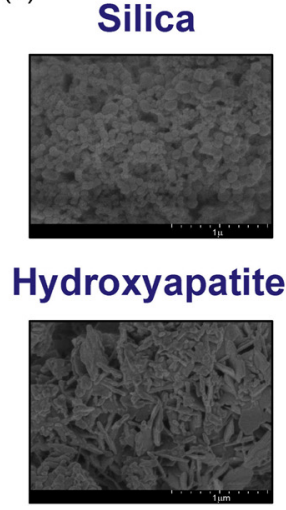
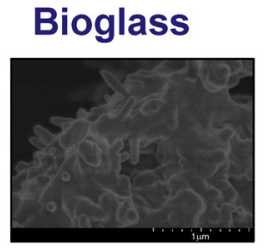

$\alpha-\mathrm{TCP}$

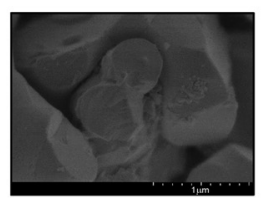

(b)

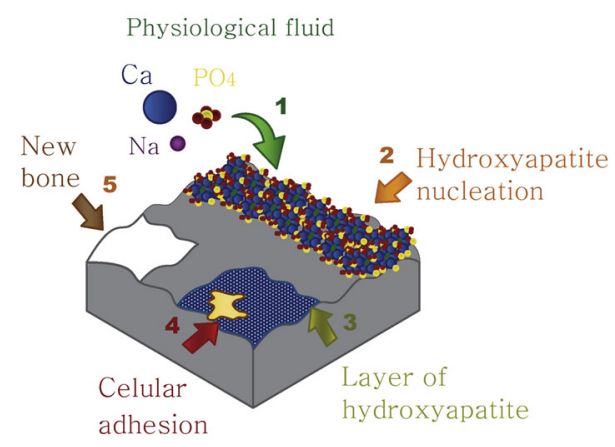

Fig. 5. a) Surface morphology of bioactive ceramics used for bone regeneration and b) New bone formation process over a bioactive ceramic material. 


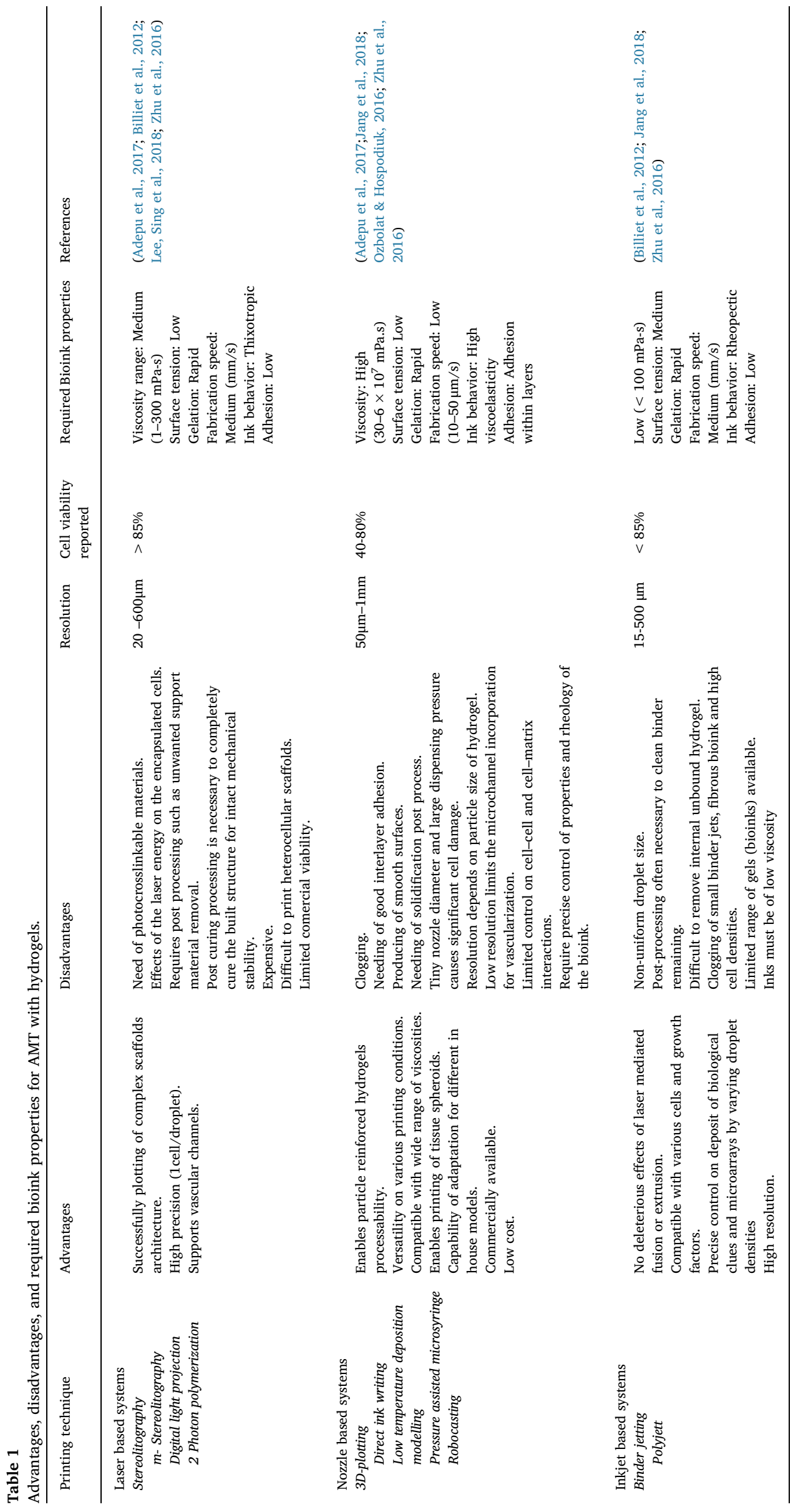




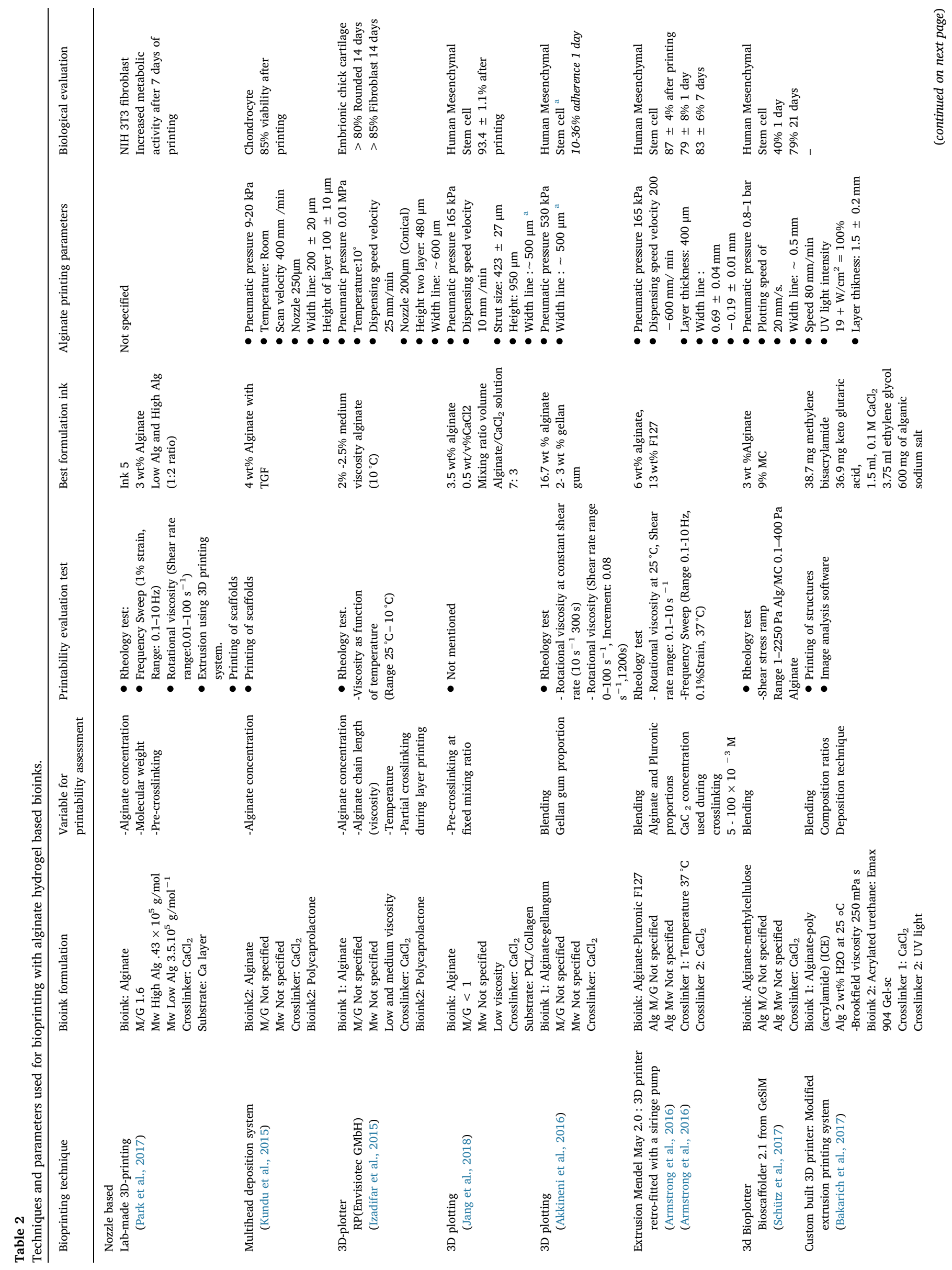




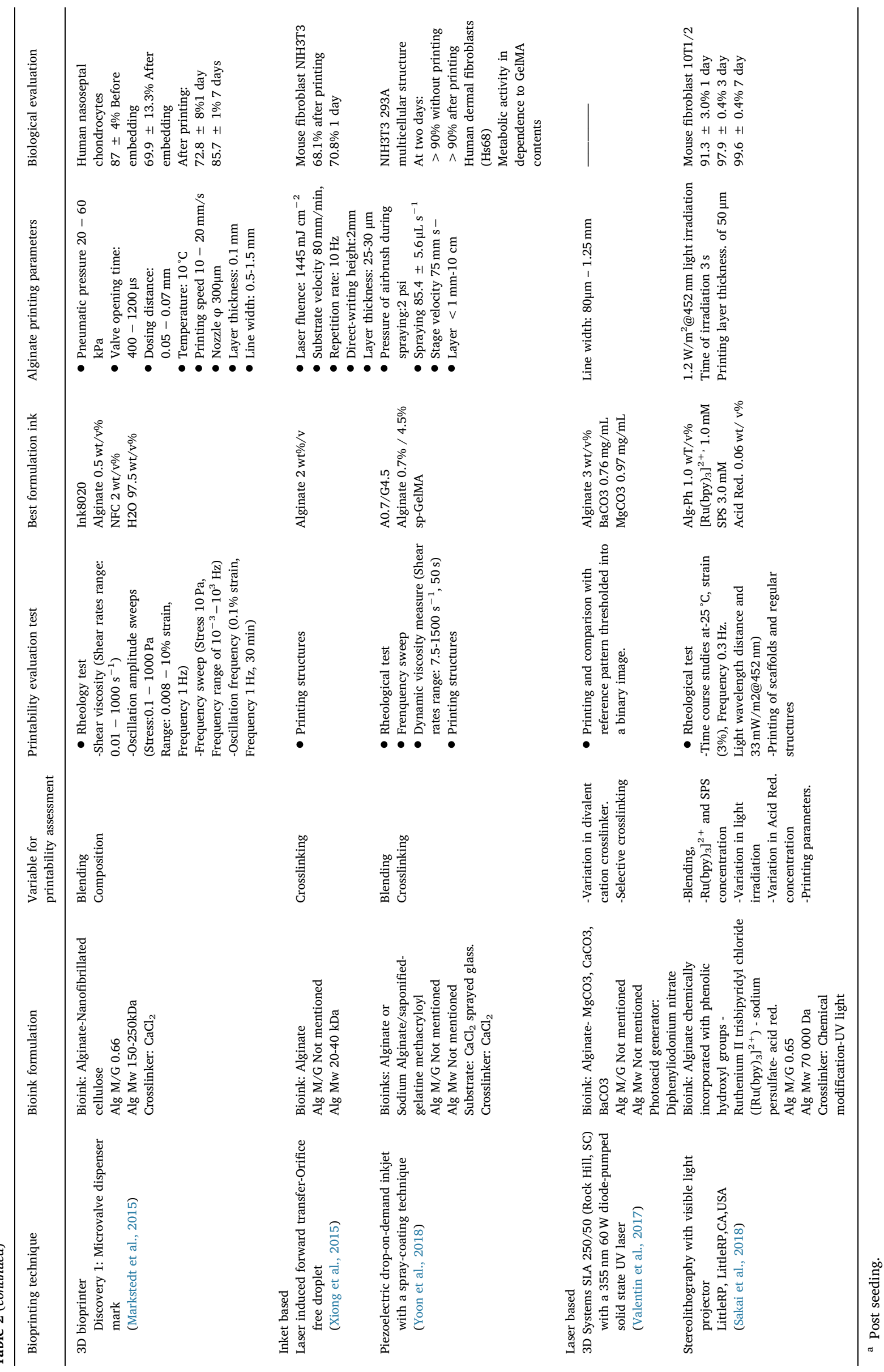


(a)

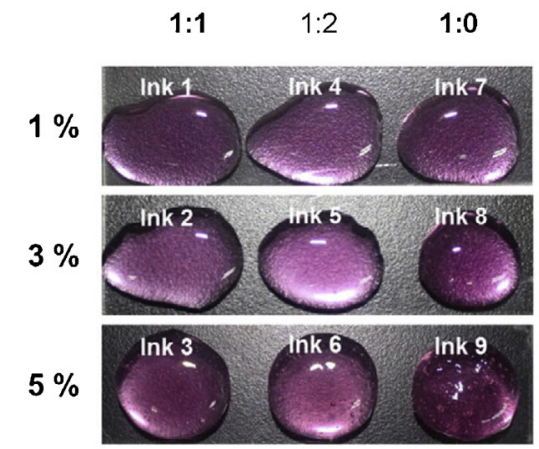

(b)
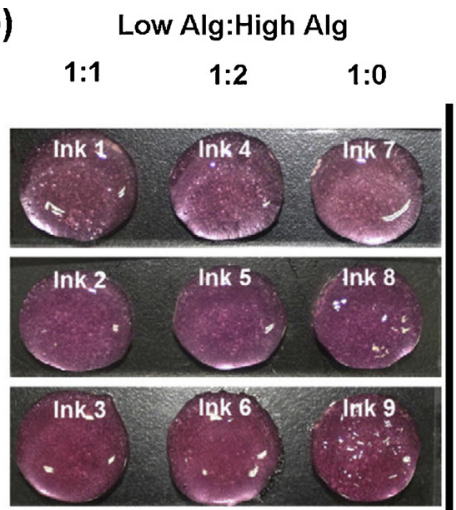

(b) without calcium ion addition.
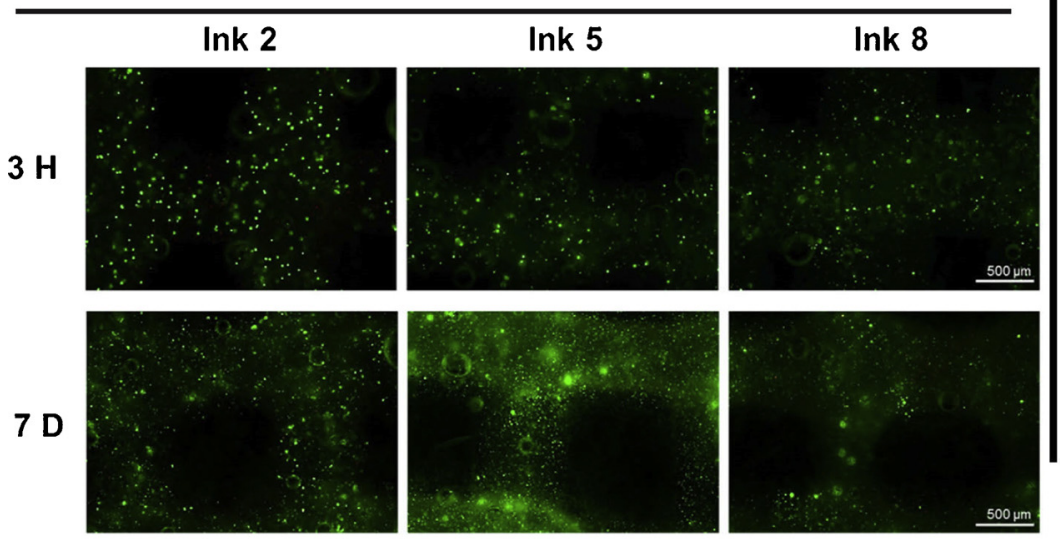

Fluorescence live/dead images of cells printed in the alginate bioinks (Ink 2, Ink 5 , and Ink 8 ) after $3 \mathrm{~h}$ and 7 days of culture. (Scale bar is $500 \mu \mathrm{m}$ ).

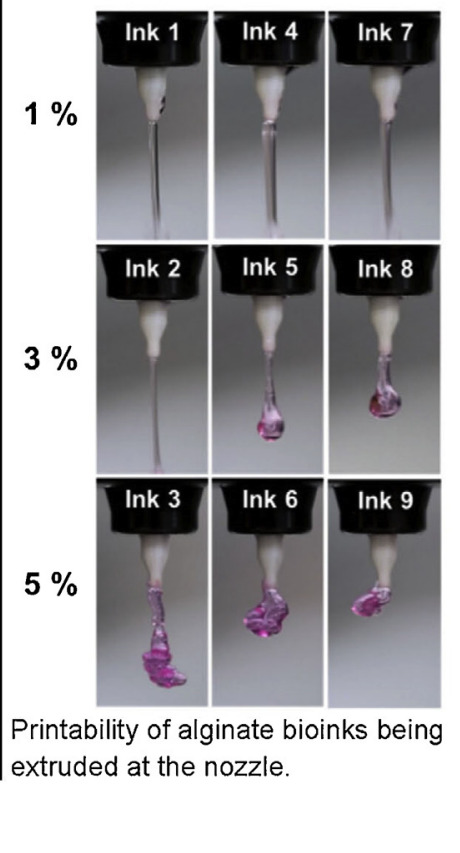

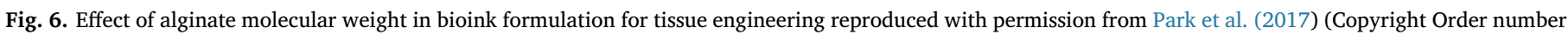
4587600358396).

increased for lower molecular weights. The authors discussed that high molecular weights produce dense networks and hinder mass transfer between the medium and the scaffolds reducing the viability and proliferation of encapsulated cells.

Kundu, Shim, Jang, Kim, and Cho (2015) developed cell-encapsulated constructs using alternated layers of polycaprolactone-alginate hydrogel (4 or $6 \mathrm{wt} \%$ ) with and without transforming growth factor (TGF- $\beta$ ). In this work, a multihead deposition system for cartilage tissue engineering was used, observing the difficulty of fabricating welldefined structures without polycaprolactone layers for low viscosity alginate solutions (concentrations $<4 \mathrm{wt} \%$ ). Also, constructs made of $4 \mathrm{wt} \%$ of alginate with TGF induced better cartilaginous tissue formation in vitro than constructs with $6 \mathrm{wt} \%$ alginate with and without TGF. Finally, it was observed that after printing, the cell viability varies with dispensing pressure, and nozzle diameter.

Izadifar, Chang, Kulyk, Chen, and Eames (2015) analyzed alginate bioinks viscosity as function of temperature and polymer viscosity for low-concentrated alginate formulations. The authors find out that bioinks prepared with $2 \% \mathrm{w}$ concentration of "medium viscosity alginate" exhibited higher viscosity than formulations having higher concentration of "low viscosity alginate" in a range of temperature from $10^{\circ} \mathrm{C}$ to $25^{\circ} \mathrm{C}$. 3D hybrid constructs of PCL strands with channels of cell-loaded alginate hydrogels were bioprinted. Hybrid constructs reported cell viability $>80 \%$ for embryonic chick cartilage cells at 14 days.

Hence, using alginate bioinks with high concentrations (i.e.3.5-10 wt/v\%) improves resolution printability and the material structural stability but limits cytocompatibility (Bohari, Hukins, \& Grover, 2011; Izadifar et al., 2015; Park et al., 2017).

In another approach, alginate blending can also modify alginate hydrogel processability. In 2016, Akkineni et al. (2016) developed highly concentrated alginate-gellan gum structures through 3D plotting. The gellan gum, decreases initial injectability of the bioink, but increases the shear thinning behavior and improves the shape fidelity of printed alginate scaffolds. Armstrong, Burke, Carter, Davis, and Perriman (2016) bioprinted several arrays of rectangular prisms and also anatomical structures of bone and cartilage 3D constructs based on a formulated bioink of sodium alginate and a poloxamer as a sacrificial guest for porosity improvement. Shear thinning properties of the bioink were optimized through the pluronic constituent, and the fidelity of the printed constructs ensured by alginate proportion and post-crosslinking with $\mathrm{CaCl}_{2}$. The evaluation of hMSC-laden 3D architectures showed high cell viability over 10 days, and osteoblastic and chondrocyte differentiation over five weeks. Schütz et al. (2017) improved the printability of a $3 \mathrm{wt} \%$ alginate based bioink by combining with MC during fabrication. The results indicate that the mixture of 1:3 ratio of alg:MC allowed a precise strand deposition, forming stable scaffolds with a regular vertical macropore structure with improved compressive modulus. The suitability of the alginate/MC blend for cell embedding was evaluated by incorporation mesenchymal stem cells during scaffold fabrication showing high viability after 3 weeks since scaffold fabrications and cell cultivation.

Di Giuseppe et al. (2018) developed Alg-Gel blends varying the individual constituent concentrations for study of printability, print accuracy, compressive behavior and viability of encapsulated mesenchymal stem cells in bioprinted constructs. Higher concentrations of both alginate and gelatine resulted in printable bioinks with an optimal crosslinking time of $15 \mathrm{~min}$ in calcium chloride to improve stability per layer. In addition, the blends with $7 \%$ Alg- $8 \%$ Gel, yields high 
printability, mechanical strength, stiffness, and cell viability after printing. However, the compressive behavior of Alg-Gel decreases rapidly over time and especially at $37^{\circ} \mathrm{C}$.

As were mentioned before, alginate bioinks are primary processed by nozzle based techniques. However, several authors have tried to take advantage of the benefits of alginates for other bioprinting technologies. As consequence, according to the printing technique, additional parameters must be contemplated for successful bioprinting of alginate bioinks.

An example is observed in the work from the group of Xiong, Zhang, and Huang (2015), Yan, Huang, and Chrisey (2012), who study the feasibility and printing quality of laser printing through the variation of alginate concentration and operating conditions such as laser fluence, laser spot size, crosslinking solution and downward movement step size of the receiving platform. In this series of works, straight and Y-shaped tubes were fabricated with high and low alginate concentrations (2 and $8 \mathrm{wt} / \mathrm{v} \%)$. Cellular constructs printed with $8 \mathrm{wt} / \mathrm{v} \%$ demonstrated high shape fidelity but constructs with low alginate concentrations $(2 \mathrm{wt} / \mathrm{v}$ $\%)$ resulted in not well-defined structures with cell viability around $70 \%$ after $24 \mathrm{~h}$ of printing. Hence the author mentioned the necessity to improve the jet and droplet formation processes and bioink printability in order to reach high quality of printed cellular constructs.

Valentin et al. (2017) printed microfluidic channels of patterned alginate by stereolithography in order to developed sacrificial alginate templates within agarose hydrogels. The 3D alginate hydrogels were printed through the selective illumination of photoacid generators in the presence of insoluble salts with divalent cation ( $\mathrm{Ca}, \mathrm{Ba} \mathrm{Mg}$ ), provoking cation dissociation and alginate crosslinking in specific patterns. Also, degradability with ethylene-diamine-tetra acetic acid (EDTA) and printing fidelity of the bioink by variation of the cations sources concentration were studied. In a study of the patterning of alginate microstructures with variable height, the authors use a "bottom-up" printing stereolithography, in which alginate precursor solution was added layer by layer, and then photopatterned. Following photopatterning, the stepped structure was washed and post-cured with $\mathrm{CaCl}_{2}$, obtaining a vertical spatial resolution around of $250 \mu \mathrm{m}$. Since alginate patterns act as sacrificial template, no cells were loaded during the SLA printing. However, cell viability of epithelial cells was measured over alginate microstructures with low-cytotoxicity results.

Sakai et al. (2018) developed mouse fibroblast-laden constructs of chemically modified alginate by stereolithographic printing, equipped with a visible light projector for crosslinking. The formulated bioink consist in alginate chemically incorporated with phenolic hydroxyl groups, a solution of ruthenium II trisbipyridyl chloride ([Ru $\left.(\text { bpy })_{3}\right]^{2+}$ ), sodium persulfate, and acid red as scattered light absorbent. The authors varied components concentration and light irradiation intensity over the formulation in order to obtain an effective bioink for stereolitography. In addition, parameters as layer thickness and scattered light absorbent were varied in order to obtain bioprinted structures with high shape fidelity. The results show that depending on the printing parameters, not all the designed structures of hydrogel constructs could be achieved, showing the strong influence of the printing parameters on the final properties of the construct. Defined rectangular prism-shaped hydrogels containing a perfusable helical lumen (diameter of $1 \mathrm{~mm}$ ) structures were able to form printing layers of $50 \mu \mathrm{m}$ thickness by irradiating light at $1.2 \mathrm{~W} / \mathrm{m}^{2} @ 452$ for $3 \mathrm{~s}$.

Zhu et al. (2018) evaluated alginate in a templating strategy using different bioinks composed of bio-macromolecular components and alginate as temporal structural support to stabilize the shape of the construct during bioprinting. In this work, the authors used a core-sheath coaxial microfluidic printhead, and the alginate component of the bioink was delivered through the core flow, with immediate crosslinking by $\mathrm{Ca}^{2+}$. Subsequently, crosslinking of the bio-macromolecular component is achieved, whether photochemically (e.g. for GelMA), chemically (i.e. for gelatin), or physically (e.g. for collagen) and finally, alginate is selectively removed through dissociation.
Biocompatibility was tested with HepG2/C3A hepatocytes, hMSCs, and MDA-MB-231 breast cancer cells for GelMA -Alg, Gelatin-alginate and collagen-alginate systes respectively, obtaining cell viability $>85 \%$ in all the printed scaffolds. However, poor printability was observed in all the bioinks, directly related to the relatively low viscosity of the components.

At this point, something to remark is that alginate bioinks usually need a partial crosslinking during or prior the construct manufacturing with the purpose of granting adequate viscosity to the material for the AMT processing (Ahn, Lee, Bonassar, \& Kim, 2012; Izadifar et al., 2015; Jang, Ahn, Yang, \& Kim, 2016). Although this process has proven to not be cytotoxic, it is an additional variable to be considered in the generation of alginate bioinks (Jang et al., 2016)

Otherwise, it has to be considered that regardless of the printability of alginate bioink, the final scaffold must meet the specific requirements of the desired application (Gopinathan \& Noh, 2018). Alginate hydrogels do not usually meet the mechanical properties required for their application as bone substitutes; the stiffness of the bone during the elastic deformations ranges between $15-25 \mathrm{GPa}$, whereas alginates is lower: 150-550 kPa (Axpe \& Oyen, 2016). Section 2.3 offer us a general overview of the injectable composites alginate-ceramic studied in order to overcome this limitation, however Section 3.2 analyses some interesting works developed related with manufacturing by 3D bioprinting.

\subsection{D-printing of alginate hydrogel-ceramic composites for bone engineering}

It has been stated that the printability of hydrogel-ceramic composites requires stimuli-dependent viscosity which usually involve changes in temperature and shear thinning to prevent the nozzle from clogging and to maintain the intended shape after printing (Wang, Jiang, Zhou, Gou, \& Hui, 2017). The addition of ceramic based nano or microparticles as rheology modifiers often interrupt the crosslinking of hydrogels and decreases the materials printability. In addition, the incorporation of these additives may decrease the accuracy of printed scaffolds due to an increase in nozzle size or it can make the resulting material completely unusable. Nevertheless, some studies have tried to achieve the successful construction of high fidelity scaffolds through 3D-printing of hydrogel-based materials incorporating inorganic phases (Jang et al., 2018).

A particle-reinforced hydrogel composite is often formed from ex situ process in which the pre-formed particles are dispersed into a hydrogel-forming liquid to be used for 3D printing. This approach allows excellent control over the quantity of incorporated particles and facilitates the study of the optimal experimental conditions. Most particlereinforced hydrogel composites are fabricated with this approach (Jang et al., 2018).

Luo, Wu, Lode, and Gelinsky (2012) combined alginate paste scaffolds with mesoporous bioglass to study the effect in the final properties for scaffolds generated by 3D plotting. The MBG/alginate pastes showed good processability during 3D plotting. Stable MBG/alginate composite scaffolds with controllable architecture were obtained. In addition, scaffolds containing $50 \mathrm{wt} \%$ of mesoporous BG showed higher compressive strength and compressive modulus in comparison to pure alginate scaffolds. Values increased from $\sim 0.7 \mathrm{MPa}$ to $\sim 1.5 \mathrm{MPa}$ and from $\sim 2 \mathrm{MPa}$ to $\sim 6 \mathrm{MPa}$. Higher apatite mineralization and cytocompatibility were observed for scaffolds containing mesoporous BG, particularly for $30 \mathrm{wt} \%$ and $50 \mathrm{wt} \%$ MBG-containing composite scaffolds. Wang et al. (2014) investigated the effect of bioglass nanoparticles $(\sim 55 \mathrm{~nm})$ addition in alginate/gelatin hydrogel matrices loaded with SaOS-2 cells using a 3D-Bioplotter. In this work the materials were mixed with polyphosphates (polyP) or biosilicas in order to increase cell proliferation. During the incubation periods (3 and 5 days), bioglass-reinforced alginate/gelatin hydrogel composites showed significant enhancement of proliferation and mineralization of bioprinted SaOS-2 cells. On the other hand, it was noticed that the 
materials containing polyPNCa ${ }^{2+}$-complex, silica, biosilica or poly$\mathrm{PNCa}^{2+}$-complex together with biosilica, did not significantly change the growth potency of the cells. No evaluation of mechanical properties is reported in this work.

Liu et al. (2019) fabricated bioactive sodium alginate/hydroxyapatite (SA/HAp) porous gel scaffolds by 3D printing. In this work an internal pre-crosslinking method of the slurry is achieved through the release of $\mathrm{Ca}$ ions from hydroxyapatite via $\mathrm{D}$-gluconic acid d-lactone (GDL). This formulation increases the viscosity of the SA/HAp slurry (33 $800 \mathrm{mPa} \cdot \mathrm{s}$ to $464000 \mathrm{mPa} \cdot \mathrm{s}$ ) improving the printability of the ink and the shape retention of the printed structure. Subsequently, the printed scaffolds were crosslinked in calcium chloride solution and then a post treatment of freeze-drying applied to obtain the SA/HAp porous scaffolds. Hydroxyapatite content and immersion times of the scaffolds in calcium chloride solution increased the degree of crosslinking of the alginate and decreased the porosity. Soaking for $5 \mathrm{~h}$ resulted in higher compression stress $(968 \mathrm{kPa})$ than non-soaking $(\sim 143 \mathrm{kPa})$ or soaking for $11 \mathrm{~h}(\sim 221 \mathrm{kPa})$. Also the compression stress of the scaffolds increased from $143 \mathrm{kPa}$ ( $1 \mathrm{wt} \% \mathrm{HAp}$ containing ink) to $1215 \mathrm{kPa}$ at $65 \%$ strain (7 wt \% HAp containing ink). Higher cellular densities were observed for materials with a 3 to $5 \mathrm{wt} \%$ of HAp. The authors concluded that the addition of HAp increased cell adhesion and proliferation respect to sodium alginate hydrogels. However, HAp addition also yielded more dense scaffolds network, and for longer periods the quicker swelling and degradation in water, decreased the space for cell growth and proliferation.

Recently, $\mathrm{SiO}_{2}$ nanoparticles were loaded into alginate-gelatin composite hydrogels chemically crosslinked with $\mathrm{CaCl}_{2}$ solution for the printing of an irregular specific defect using a BIOBOT (Allevi) 3D printer (Roopavath, Soni, Mahanta, Deshpande, \& Rath, 2019). The effect of $\mathrm{SiO}_{2}$ nanoparticles on the viscosity, degradation, compressive modulus (MPa), biocompatibility and osteogenic ability post-seeding were evaluated on lyophilized scaffolds. The addition of $\mathrm{SiO}_{2}$ nanoparticles into the hydrogel system at 2.5 and $5 \mathrm{wt} \%$, increased the viscosity of the hydrogel ink, which allowed the printability of scaffolds. Compressive modulus $(\mathrm{MPa})$ increased from $32.57 \pm 0.98 \mathrm{MPa}$ to $49.18 \pm 1.64 \mathrm{MPa}$ for these composite scaffolds, but swelling and degradation properties were inhibited. The metabolic activity of the cells in all sample groups increased from day 1 to day 21 with a marked dependence on the micro porosity of the structures. Also higher ALP activity was observed for higher concentrations of $\mathrm{SiO}_{2}$ nanoparticles.

In other approach, a 3D printed composite based on alginate and in situ formed calcium phosphate (CP) was presented by Egorov et al. (2016). The CP phase was formed upon mixing the polymer slurry containing ammonium hydrogen phosphate with a calcium chloride aqueous solution during the printing process. The compressive strength of composite hydrogels was gradually increased from 0.45 to $1.0 \mathrm{MPa}$, with increasing concentrations of alginate from 0.25 to $4 \mathrm{wt} \%$. However, overall values of compressive strength for 3D printed scaffold were relatively low due to the weak bonding between printed layers, which is the major limitation of the in situ particle incorporation approach for composite hydrogel systems.

Recently, a variety of nanosilicate clay (Laponite) has been incorporated into hydrogel composites in order to take advantage of the clay self-supporting capacity for 3D extrusion of hard tissues. Jin, Liu, Chai, Compaan, and Huang (2017) used Laponite RD and XLG in a hydrogel printing approach without any supporting bath. In this work, the nanoclay was mixed with three types of hydrogels including alginate, diacrylate poly(ethylene glycol) (PEGDA) and gelatin, and further extruded in air by direct ink writing with the appropriate crosslinking method. The addition of Laponite increased the Young's modulus (from $\sim 2.5$ to $\sim 15 \mathrm{kPa}$ ) of extruded scaffolds, and it also adjusted the degradation rates. However, only the cytocompatibility of PEGDA-Laponite was confirmed using fibroblast cell adhesion and proliferation experiments.

In addition, the possibility of bioprinting and simultaneous growth factor delivery from hydrogel-nanoclay composites was verified by Ahlfeld et al. (2017). Laponite XLG was blended with compositions of alginate-methylcellulose hydrogels and loaded with human telomerase reverse transcriptase mesenchymal stem cells (hTERT-MSC) for 3D plotting. For the release test, hydrogel composites were also loaded with bovine serum albumin (BSA) and vascular endothelial growth factor (VEGF). The obtained printed scaffolds showed high shape fidelity with cell viability of $(\approx 70 \%-75 \%)$ after 21 days. Continuous release of BSA and VEGF from the hydrogel composite scaffolds was observed, even after 21 and 7 days, respectively. Mechanical properties of scaffolds decreased over the time test when they were stored under cell culture conditions. Young's modulus from $176 \pm 8.9 \mathrm{kPa}$ to $28.6 \pm 9.3 \mathrm{kPa}$ and compressive strength from $40.8 \pm 4.7 \mathrm{kPa}$ to $1.6 \pm 0.4 \mathrm{kPa}$. Nevertheless, shape of the plotted constructs was preserved even over longer cultivation periods.

It is notorious that alginate-ceramic composites capability as bioinks are limited by their processability at high ceramic loading which lead to fabricate structures only with low ceramic contents and thus, low mechanical performance. In addition alginate-ceramic composites hardly can be used as cell-loaded matrices themselves. Therefore, other approximations have to be implemented in order to overcome these drawbacks.

An interesting proposal was developed by Raja and Yun (2016) through a combination of cement chemistry, a dual paste-extruding deposition (PED) and cell printing. In this work, a core/shell structured scaffold composed of calcium deficient hydroxyapatite (CDHA) and a pre-osteoblast MC3T3-E1 laden alginate hydrogel was designed. A 3Dprinting design consisted in a metallic core nozzle covered by an outer nozzle extruding the core ceramic precursor ( $\alpha$-TCP) and the shell laden alginate by mechanical and pneumatic pressure simultaneously. The core diameter and the shell thickness were controlled by parameters as mechanical and pneumatic pressure, nozzle size, printing speed and screw speed. Also parameters like alginate and crosslinker concentrations, setting time, and culture media were evaluated. The optimal development of the structures was as follows: the $\alpha$-TCP paste and the cell-loaded alginate were extruded by the 3D printing system with different thickness of core-shell. Then, the printed structures were immersed in $2.5 \mathrm{wt} \% \mathrm{CaCl}_{2}$ solution for $30 \mathrm{~min}$ to completely crosslink the hydrogel shell. The setting of the $\alpha$-TCP core was initiated by immersing the scaffold in PBS at $37^{\circ} \mathrm{C}$ during $6 \mathrm{~h}$, and later replaced with cell medium, which continued the setting process and also provided nutrition for the printed cells until $72 \mathrm{~h}$. Structures with compressive strength and modulus of $3.2 \mathrm{MPa}$ and $10.92 \mathrm{MPa}$ (in wet) respectively with less compression deformation $(32 \% \mathrm{v})$ when compared with pure alginate scaffolds $(50 \mathrm{v} \%)$ were obtained. In addition, the cells incorporated into the shell remained alive throughout the 3D scaffold for 35 days. However no changes in cell dimensions was observed in the hydrogel shell, which is proposed being solved by the addition of cell adhesion motifs to the alginate precursor.

\section{Remarks and new directions in alginate based bone engineering}

The healing of bone defects has witnessed a paradigm shift from synthetic implants and tissue grafts to a bone tissue engineering (BTE) approach that incorporates biodegradable composite scaffolds with biological cells. Alginates offer properties that have been exploited for the generation of materials for medical devices and treatments, where their gelling capacity, low toxicity, high availability and low cost stand out. Alginate hydrogels specifically provide an appropriate niche, scaffolding and environmental matrix for cell loading. In addition, their inherent ionic crosslinking make them injectable which offers advantages over solid scaffolds such as the possibility of using a non-invasive approach (Tan \& Marra, 2010). However, as described in former sections, several aspects are hindering the development of suitable bone constructs to implement bone regeneration. We summarized them in 
this section plus adding the new possible developments that in the opinion of the authors may lead to overcome current status.

The factors that avoid taking full advantage of the alginate properties for bone regeneration can be grouped into three categories: the available information from published papers; the lack of further/more innovative chemical modifications; the need for new technological developments, and the absence of protocols to assess printability of different bioinks.

The use of different alginate types, and the limited alginate specifications reported, can be the reason behind the lack of progression from previously published papers. Alginates can be extracted from different sources and have different $M$ and $G$ contents along with the length of each block, influencing the properties of the material as explained before. Alginates are also available in a wide range of molecular weights and viscosities. Since the extraction process, source and molecular weight of the alginate precursor directly influence the properties of the final material, it is necessary that the percentage of $M$ and $G$ blocks and the length of the blocks together with the molecular weights are specified in each paper to be related with the obtained properties.

Putting this aside, there are other drawbacks inferred to alginate hydrogels related with the assayed chemical modifications. Low structural integrity associated to ionic crosslinking has been deal with by alginate chemical modifications and the blending with other compounds through covalent bonding. Following this trend, modified alginate backbone with photocrosslinkable groups for covalent crosslinking via UV/Vis radiation have been developed (Chen, Kawazoe, \& Ito, 2018; Jeon et al., 2012; Lewandowska-Łańcucka et al., 2017; Yuan et al., 2017). These materials are reported to be injectable, however, this method may introduce cytotoxicity from the radicals generated by dissociation of the photoinitiators, local inflammation due to unreacted double bonds, lack of control over the crosslinking kinetics and may yield materials with oxygen inhibition. Thus, the searches of controlled photocrosslinking conditions or the utilization of mechanisms that inhibit the deleterious effect of the chemicals used have not been provided yet.

Alginate controlled oxidation and its assembly with amino groups containing compounds, such as gelatin (Balakrishnan et al., 2014; Reakasame \& Boccaccini, 2017; Sarker et al., 2014), yield faster degradation rate, injectability and better cellular response (Sarker et al., 2017) than native alginate. Promising alternatives include Mucilage, a hydrocolloid containing high molecular and low molecular weight fractions (Stintzing \& Carle, 2005) where high molecular weight fractions are made of pectin. The advantage of these groups is their ability of promoting cellular adhesion and they also have a more controllable degradation kinetics in comparison with alginates (Archana et al., 2013; Chen, Ni et al., 2017; de Souza, de Souza, Drouin, Mantovani, \& Moraes, 2019).

A particularly inspiring challenge continues to be the guiding of mineralization through biomimetic route approaches. Organic matrices offer this possibility based on tuneable organic-inorganic interactions (Palmer, Newcomb, Kaltz, Spoerke, \& Stupp, 2008) works with amelogenin (Ruan, Zhang, Yang, Nutt, \& Moradian-Oldak, 2013) or gelatin (Busch, Schwarz, \& Kniep, 2001) have enabled the growth of aligned apatite nanocrystals directly on enamel surface. Nonetheless, the development of organized apatite nanocrystals with a distinctive hierarchical order from the crystallographic-, nano-, micro-, and macroscale, is still an exciting, yet unattained, goal.

Therefore, further attempts need to be implemented to optimize the osteoinductive behavior. Among the promising attempts, the conjunction with growth factors, bone cells or bioactive materials have been assayed as described in former sections, where hydroxyapatite is one of the most used ceramics in order to promote the attachment of cultured osteoblasts in multiple polymers and it is commonly used to improve mechanical properties (You, Chen, Cooper, Chang, \& Eames, 2018). However, regarding the alginate-hydroxyapatite system, the difficulties of hydroxyapatite dispersion, the viscosity of the alginate and the negative charge density of both, partially prevents their interaction. This hinders their processing together, especially for injectable materials (Bao, Senos, Almeida, \& Gauckler, 2002; Jewad, Bentham, Hancock, Bonfield, \& Best, 2008; Lee, Choi, Kim, \& Lee, 2006). An alternative breakthrough may come from the surface functionalization of hydroxyapatite with alginate or OA. Hydroxyapatite, due to its ion exchange capacity, adsorption capacity and acidic properties, can be used as effective sorbents and carriers for polymers (Russo et al., 2014). These properties together with the alginate ability to encapsulate cells and growth factors could be a promising approach to encourage osteoinduction from simultaneous fronts.

In addition, alginate hydrogels do not reach the structural properties required to function as mechanical support in bone tissue engineering. The attempts made with alginate-cement compositions report higher mechanical properties than alginate hydrogels (compressive strength 25-70 MPa, Young modulus 90-130 MPa), but still far from the average mechanical properties of human bone; cortical bone: compression strength $200 \pm 36 \mathrm{MPa}$, elastic modulus on compression $23 \pm 4.8 \mathrm{GPa}$, cancellous bone-compression strength $1.5-38 \mathrm{MPa}$, elastic modulus on compression 10-1570 MPa (An \& Draughn, 1999). From engineering materials perspective, organic-inorganic hybrid materials may be a promising alternative to this drawback. Since the components are bonded at molecular level, stable materials with higher integrity could be obtained (Follmann et al., 2017). Among them, promising materials based on the organic-inorganic interaction are the silica-alginate compositions. Silica hybrid materials can be obtained by sol-gel technique introducing a polymer prior to gelation, and although this kind of materials has been prepared over nearly 15 years, their combination with polysaccharides for regeneration treatments has been barely explored. Silica is characterized by a tighter interface with polymer matrices in composite and hybrid materials, providing polymer scaffolds with biomineralization abilities and increasing the overall stiffness (Sowjanya et al., 2013). Since the mechanical properties of hybrid materials are strongly dependent on the intensity of interactions between the organic and the inorganic components, increasing the interfacial interactions through the covalent coupling between modified alginate and silica may yield the required mechanical properties. Covalent bonding between the co-networks can be achieved by functionalizing the polymer with a silane coupling agent for further bonding by sol-gel silica (Hosoya et al., 2004).

There is still a need to optimize the final properties obtained with alginate based materials for their potential application in bone engineering. From the point of view of the authors, bioprinting could be an adequate tool for tailoring the properties of alginate based hydrogels to the requirements of bone regeneration. The capacity of designing structures layer by layer and the ability to vary their processing conditions with a finer control over the principal alginate manufacturing conditions (addition of components, crosslinking agent concentration, reinforcement disposition, dispersant, temperature, between others), give possibilities to understand the real capacity of the material for its application.

Hence, with the knowledge of the response capacity of the material to certain conditions possible to generate technologies to stimulate the desired properties or to process these materials into challenging structures. An example is observed in work of Bakarich et al. (2017), who developed a custom built 3D printer equipped with an extrusion based deposition system in order to print blends of two components with gradient structures and spatially varying material properties. Alginate/ poly(acrylamide) ionic covalent entanglement (ICE) and commercial acrylated urethane UV-curable adhesive (Emax $904 \mathrm{Gel}-\mathrm{SC}$ ) were used simultaneously as bioinks. The results show that the digitally controlled print heads can accurately dispense the two inks in a programed ratio to form scaffolds with gradient structures, where varying the ratio in which the two inks are dispensed allows to control the swelling ratio and mechanical properties of the printed composites. Bone tissue regeneration probably requires a higher mechanical support than alginate 
hydrogels can provide by themselves to achieve suitable devices with the required shape and size. The Initial support can be provided by simultaneously printing a compatible scaffold that fulfils the mechanical requirements by a combination of printing technologies. However alginates should not be discharged as bioinks because, and as explained along the review "alginate hydrogels stand out due to their gelling facility, ability to encapsulate cells and bioactive molecules, and shear thinning behavior, allowing their use in combinational strategies"

From the bioink perspective, it has been established that Printability is directed by the rheological properties of materials and must be adjusted to the specific fabrication process to generate constructs with high shape fidelity (Paxton et al., 2017).

Rheological analysis should help to accelerate the development of printable materials as shown by several groups (Malda et al., 2013). However, after this bibliographic revision, it was perceived that comparable, reproducible and efficient characterization techniques are still to be established. Furthermore, shape fidelity has been prevalently assessed only qualitatively through visual inspection after printing hampering the study of other parameters such as filament collapsing.

This scarcity of data does not allow a proper comparison between different bioinks or even between studies involving the same bioink, leading to process materials based only on qualitative parameters yielding unexpected results. Only a few works propose reproducible methods for bioinks evaluation (Paxton et al., 2017), Ribeiro et al. (2017), Valentin et al. (2017), and could set up the basis for a reproducible universal testing methodology of printable bioinks.

Thus, the generation of technologies that suits to the specific needs for processing a material could be an effective response to the "bottleneck" in the development of applicable bone substitutes.

Finally, it must be considered that when trying to introduce novel materials into clinical practice, cooperation of interdisciplinary groups is necessary; research scientists, industry, academia, government, nonprofit organizations, clinical investigators, patients, payers, and regulators should all be involved. Therefore, incentivizing the communication channels is essential in order to overcome the barriers that are avoiding tissue engineering to provide successful products to the market.

\section{Declaration of Competing Interest}

None.

\section{Acknowledgements}

Funding: This work was supported by Instituto Politécnico Nacional, México (IPN-SIP 20196660) and AEI/FEDER, UE (DPI2017-90147-R). Financial support from CONACYT scholarship is acknowledged.

\section{Appendix A. Supplementary data}

Supplementary material related to this article can be found, in the online version, at doi:https://doi.org/10.1016/j.carbpol.2019.115514.

\section{References}

Adepu, S., Dhiman, N., Laha, A., Sharma, C. S., Ramakrishna, S., \& Khandelwal, M. (2017). Three-dimensional bioprinting for bone tissue regeneration. Current Opinion in Biomedical Engineering, 2, 22-28.

Ahlfeld, T., Cidonio, G., Kilian, D., Duin, S., Akkineni, A., Dawson, J., ... Gelinsky, M. (2017). Development of a clay based bioink for 3D cell printing for skeletal application. Biofabrication, 9(3), 034103.

Ahn, S., Lee, H., Bonassar, L. J., \& Kim, G. (2012). Cells (MC3T3-E1)-laden alginate scaffolds fabricated by a modified solid-freeform fabrication process supplemented with an aerosol spraying. Biomacromolecules, 13(9), 2997-3003.

Akkineni, A. R., Ahlfeld, T., Funk, A., Waske, A., Lode, A., \& Gelinsky, M. (2016). Highly concentrated alginate-gellan gum composites for 3D plotting of complex tissue engineering scaffolds. Polymers, 8(5), 170.

An, Y. H., \& Draughn, R. A. (1999). Mechanical testing of bone and the bone-implant interface. CRC Press.
Ansari, S., Diniz, I. M., Chen, C., Aghaloo, T., Wu, B. M., Shi, S., ... Moshaverinia, A. (2017). Alginate/hyaluronic acid hydrogel delivery system characteristics regulate the differentiation of periodontal ligament stem cells toward chondrogenic lineage. Journal of Materials Science: Materials in Medicine, 28(10), 162.

Archana, D., Upadhyay, L., Tewari, R., Dutta, J., Huang, Y., \& Dutta, P. (2013). Chitosanpectin-alginate as a novel scaffold for tissue engineering applications.

Armstrong, J. P., Burke, M., Carter, B. M., Davis, S. A., \& Perriman, A. W. (2016). 3D bioprinting using a templated porous bioink. Advanced Healthcare Materials, 5(14), 1724-1730.

Ashammakhi, N., Hasan, A., Kaarela, O., Byambaa, B., Sheikhi, A., Gaharwar, A. K., Khademhosseini, A. (2019). Advancing frontiers in bone bioprinting. Advanced Healthcare Materials 1801048.

Awad, H. A., Wickham, M. Q., Leddy, H. A., Gimble, J. M., \& Guilak, F. (2004). Chondrogenic differentiation of adipose-derived adult stem cells in agarose, alginate, and gelatin scaffolds. Biomaterials, 25(16), 3211-3222.

Axpe, E., \& Oyen, M. L. (2016). Applications of alginate-based bioinks in 3D bioprinting. International Journal of Molecular Sciences, 17(12), 1976.

Bai, X., Lü, S., Liu, H., Cao, Z., Ning, P., Wang, Z., \& Liu, M. (2017). Polysaccharides based injectable hydrogel compositing bio-glass for cranial bone repair. Carbohydrate Polymers, $175,557-564$.

Bajpai, M., Shukla, P., \& Bajpai, S. (2016). Ca (II) + Ba (II) ions crosslinked alginate gels prepared by a novel diffusion through dialysis tube (DTDT) approach and preliminary BSA release study. Polymer Degradation and Stability, 134, 22-29.

Bakarich, S. E., Gorkin, R., III, Gately, R., Naficy, S., in het Panhuis, M., \& Spinks, G. M. (2017). 3D printing of tough hydrogel composites with spatially varying materials properties. Additive Manufacturing, 14, 24-30.

Balakrishnan, B., \& Jayakrishnan, A. (2005). Self-cross-linking biopolymers as injectable in situ forming biodegradable scaffolds. Biomaterials, 26(18), 3941-3951.

Balakrishnan, B., Joshi, N., Jayakrishnan, A., \& Banerjee, R. (2014). Self-crosslinked oxidized alginate/gelatin hydrogel as injectable, adhesive biomimetic scaffolds for cartilage regeneration. Acta Biomaterialia, 10(8), 3650-3663.

Balakrishnan, B., Mohanty, M., Umashankar, P., \& Jayakrishnan, A. (2005). Evaluation of an in situ forming hydrogel wound dressing based on oxidized alginate and gelatin. Biomaterials, 26(32), 6335-6342.

Baniasadi, H., Mashayekhan, S., Fadaoddini, S., \& Haghirsharifzamini, Y. (2016). Design, fabrication and characterization of oxidized alginate-gelatin hydrogels for muscle tissue engineering applications. Journal of Biomaterials Applications, 31(1), 152-161.

Bao, Y., Senos, A., Almeida, M., \& Gauckler, L. J. (2002). Rheological behavior of aqueous suspensions of hydroxyapatite (HAP). Journal of Materials Science Materials in Medicine, 13(7), 639-643.

Becker, T. A., \& Kipke, D. R. (2002). Flow properties of liquid calcium alginate polymer injected through medical microcatheters for endovascular embolization. Journal of Biomedical Materials Research Part A, 61(4), 533-540.

Bidarra, S. J., Barrias, C. C., Fonseca, K. B., Barbosa, M. A., Soares, R. A., \& Granja, P. I. (2011). Injectable in situ crosslinkable RGD-modified alginate matrix for endothelial cells delivery. Biomaterials, 32(31), 7897-7904.

Bidarra, S. J., Barrias, C. C., \& Granja, P. L. (2014). Injectable alginate hydrogels for cell delivery in tissue engineering. Acta Biomaterialia, 10(4), 1646-1662.

Billiet, T. V. M., Schelfhout, J., Van Vlierberghe, S., \& Dubruel, P. (2012). A review of trends and limitations in hydrogel-rapid prototyping for tissue engineering. Biomaterials, 33(26), 6020-6041.

Bohari, S. P., Hukins, D. W., \& Grover, L. M. (2011). Effect of calcium alginate concentration on viability and proliferation of encapsulated fibroblasts. Bio-medical Materials and Engineering, 21(3), 159-170.

Bonino, C. A., Krebs, M. D., Saquing, C. D., Jeong, S. I., Shearer, K. L., Alsberg, E., ... Khan, S. A. (2011). Electrospinning alginate-based nanofibers: From blends to crosslinked low molecular weight alginate-only systems. Carbohydrate Polymers, 85(1), 111-119.

Bouhadir, K. H., Kruger, G. M., Lee, K. Y., \& Mooney, D. J. (2000). Sustained and controlled release of daunomycin from cross-linked poly (aldehyde guluronate) hydrogels. Journal of Pharmaceutical Sciences, 89(7), 910-919.

Bouhadir, K. H., Lee, K. Y., Alsberg, E., Damm, K. L., Anderson, K. W., \& Mooney, D. J. (2001). Degradation of partially oxidized alginate and its potential application for tissue engineering. Biotechnology Progress, 17(5), 945-950.

Bu, H., Kjøniksen, A.-L., Knudsen, K. D., \& Nyström, B. (2004). Rheological and structural properties of aqueous alginate during gelation via the Ugi multicomponent condensation reaction. Biomacromolecules, 5(4), 1470-1479.

Burana-osot, J., Hosoyama, S., Nagamoto, Y., Suzuki, S., Linhardt, R. J., \& Toida, T. (2009). Photolytic depolymerization of alginate. Carbohydrate Research, 344(15), 2023-2027.

Busch, S., Schwarz, U., \& Kniep, R. (2001). Morphogenesis and structure of human teeth in relation to biomimetically grown fluorapatite - gelatine composites. Chemistry of Materials, 13(10), 3260-3271.

Buwalda, S. J. B., Kristel, W. M., Dijkstra, P. J., Feijen, J., Vermonden, T., \& Hennink, W. E. (2014). Hydrogels in a historical perspective: From simple networks to smart materials. Journal of Controlled Release, 190, 254-273.

Caló, E., \& Khutoryanskiy, V. V. (2015). Biomedical applications of hydrogels: A review of patents and commercial products. European Polymer Journal, 65, 252-267.

Chan, L., Jin, Y., \& Heng, P. (2002). Cross-linking mechanisms of calcium and zinc in production of alginate microspheres. International Journal of Pharmaceutics, 242(1), 255-258.

Chen, F., Ni, Y., Liu, B., Zhou, T., Yu, C., Su, Y., ... Zhou, Y. (2017). Self-crosslinking and injectable hyaluronic acid/RGD-functionalized pectin hydrogel for cartilage tissue engineering. Carbohydrate Polymers, 166, 31-44.

Chen, G., Kawazoe, N., \& Ito, Y. (2018). Photo-crosslinkable hydrogels for tissue engineering applications. Photochemistry for biomedical applications. Springer277-300.

Chen, H., Xing, X., Tan, H., Jia, Y., Zhou, T., Chen, Y., ... Hu, X. (2017). Covalently 
antibacterial alginate-chitosan hydrogel dressing integrated gelatin microspheres containing tetracycline hydrochloride for wound healing. Materials Science and Engineering C, 70, 287-295.

Chen, Q., Zhu, C., \& Thouas, G. A. (2012). Progress and challenges in biomaterials used for bone tissue engineering: Bioactive glasses and elastomeric composites. Progress in Biomaterials, 1(1), 2.

Chung, J. H., Naficy, S., Yue, Z., Kapsa, R., Quigley, A., Moulton, S. E., ... Wallace, G. G. (2013). Bio-ink properties and printability for extrusion printing living cells. Biomaterials Science, 1(7), 763-773.

Coleman, R. J., Lawrie, G., Lambert, L. K., Whittaker, M., Jack, K. S., \& Grøndahl, L. (2011). Phosphorylation of alginate: Synthesis, characterization, and evaluation of in vitro mineralization capacity. Biomacromolecules, 12(4), 889-897.

Dalheim, M.Ø., Vanacker, J., Najmi, M. A., Aachmann, F. L., Strand, B. L., \& Christensen, B. E. (2016). Efficient functionalization of alginate biomaterials. Biomaterials, 80 , $146-156$.

de Souza, F. C. B., de Souza, R. F. B., Drouin, B., Mantovani, D., \& Moraes, Â. M. (2019). Comparative study on complexes formed by chitosan and different polyanions: Potential of chitosan-pectin biomaterials as scaffolds in tissue engineering. International Journal of Biological Macromolecules, 132, 178-189.

de Vos, P., Lazarjani, H. A., Poncelet, D., \& Faas, M. M. (2014). Polymers in cell en capsulation from an enveloped cell perspective. Advanced Drug Delivery Reviews, 67, 15-34.

Derakhshanfar, S., Mbeleck, R., Xu, K., Zhang, X., Zhong, W., \& Xing, M. (2018). 3D bioprinting for biomedical devices and tissue engineering: A review of recent trends and advances. Bioactive Materials, 3(2), 144-156.

Desai, R. M., Koshy, S. T., Hilderbrand, S. A., Mooney, D. J., \& Joshi, N. S. (2015). Versatile click alginate hydrogels crosslinked via tetrazine-norbornene chemistry. Biomaterials, 50, 30-37.

Di Giuseppe, M., Law, N., Webb, B., Macrae, R. A., Liew, L. J., Sercombe, T. B., ... Doyle, B. J. (2018). Mechanical behaviour of alginate-gelatin hydrogels for 3D bioprinting. Journal of the Mechanical Behavior of Biomedical Materials, 79, 150-157.

Ding, W., Zhou, J., Zeng, Y., Wang, Y.-n., \& Shi, B. (2017). Preparation of oxidized sodium alginate with different molecular weights and its application for crosslinking collagen fiber. Carbohydrate Polymers, 157, 1650-1656.

Follmann, H. D. M., Naves, A. F., Araujo, R. A., Dubovoy, V., Huang, X., Asefa, T., .. Oliveira, O. N. (2017). Hybrid materials and nanocomposites as multifunctional biomaterials. Current Pharmaceutical Design, 23(26), 3794-3813.

Dorozhkin, S. V., \& Epple, M. (2002). Biological and medical significance of calcium phosphates. Angewandte Chemie International Edition, 41(17), 3130-3146.

Douglas, T. E., Schietse, J., Zima, A., Gorodzha, S., Parakhonskiy, B. V., KhaleNkow, D., ... Weinhardt, V. (2018). Novel self-gelling injectable hydrogel/alpha-tricalcium phosphate composites for bone regeneration: Physiochemical and microcomputer tomographical characterization. Journal of Biomedical Materials Research Part A, 106(3), 822-828.

Dragan, E. S. (2014). Design and applications of interpenetrating polymer network hydrogels. A review. Chemical Engineering Journal, 243, 572-590.

Egorov, A. A., Fedotov, A. Y., Mironov, A. V., Komlev, V. S., Popov, V. K., \& Zobkov, Y. V. (2016). 3D printing of mineral-polymer bone substitutes based on sodium alginate and calcium phosphate. Beilstein Journal of Nanotechnology, 7, 1794.

El-Rashidy, A. A., Roether, J. A., Harhaus, L., Kneser, U., \& Boccaccini, A. R. (2017). Regenerating bone with bioactive glass scaffolds: A review of in vivo studies in bone defect models. Acta Biomaterialia, 62, 1-28.

Evangelista, M. B., Hsiong, S. X., Fernandes, R., Sampaio, P., Kong, H.-J., Barrias, C. C., .. Granja, P. L. (2007). Upregulation of bone cell differentiation through immobilization within a synthetic extracellular matrix. Biomaterials, 28(25), 3644-3655.

Falkeborg, M., Cheong, L.-Z., Gianfico, C., Sztukiel, K. M., Kristensen, K., Glasius, M., .. Guo, Z. (2014). Alginate oligosaccharides: Enzymatic preparation and antioxidant property evaluation. Food Chemistry, 164, 185-194.

Fedorovich, N. E., Wijnberg, H. M., Dhert, W. J., \& Alblas, J. (2011). Distinct tissue formation by heterogeneous printing of osteo-and endothelial progenitor cells. Tissue Engineering Part A, 17(15-16), 2113-2121.

Feng, L., Cao, Y., Xu, D., Wang, S., \& Zhang, J. (2017). Molecular weight distribution, rheological property and structural changes of sodium alginate induced by ultrasound. Ultrasonics Sonochemistry, 34, 609-615.

Fernández-Montes Moraleda, B., Román, J. S., \& Rodríguez-Lorenzo, L. M. (2013). Influence of surface features of hydroxyapatite on the adsorption of proteins relevant to bone regeneration. Journal of Biomedical Materials Research Part A, 101(8), 2332-2339.

Fernández-Montes Moraleda, B., San Román, J., \& Rodríguez-Lorenzo, L. M. (2016). Adsorption and conformational modification of fibronectin and fibrinogen adsorbed on hydroxyapatite. A QCM-D study. Journal of Biomedical Materials Research Part A, 104(10), 2585-2594.

Fonseca, K. B., Bidarra, S. J., Oliveira, M. J., Granja, P. L., \& Barrias, C. C. (2011). Molecularly designed alginate hydrogels susceptible to local proteolysis as three-dimensional cellular microenvironments. Acta Biomaterialia, 7(4), 1674-1682.

Freeman, F. E., \& Kelly, D. J. (2017). Tuning alginate bioink stiffness and composition for controlled growth factor delivery and to spatially direct MSC fate within bioprinted tissues. Scientific Reports, 7(1), 17042.

Gao, C., Liu, M., Chen, J., \& Zhang, X. (2009). Preparation and controlled degradation of oxidized sodium alginate hydrogel. Polymer Degradation and Stability, 94(9), $1405-1410$

Gombotz, W. R., \& Wee, S. (1998). Protein release from alginate matrices. Advanced Drug Delivery Reviews, 31(3), 267-285.

Gomez, C., Rinaudo, M., \& Villar, M. (2007). Oxidation of sodium alginate and characterization of the oxidized derivatives. Carbohydrate Polymers, 67(3), 296-304.

Gonzalez-Pujana, A., Orive, G., Pedraz, J. L., Santos-Vizcaino, E., \& Hernandez, R. M.
(2018). Alginate microcapsules for drug delivery. Alginates and their biomedical applications. Springer67-100.

Gopinathan, J., \& Noh, I. (2018). Recent trends in bioinks for 3D printing. Biomaterials Research, 22(1), 11.

Gothard, D., Smith, E. L., Kanczler, J. M., Black, C. R., Wells, J. A., Roberts, C. A., .. Rashidi, H. (2015). In vivo assessment of bone regeneration in alginate/bone ECM hydrogels with incorporated skeletal stem cells and single growth factors. PLoS One, 10(12), e0145080.

Grant, G. T., Morris, E. R., Rees, D. A., Smith, P. J., \& Thom, D. (1973). Biological interactions between polysaccharides and divalent cations: The egg-box model. FEBS Letters, 32(1), 195-198.

Grellier, M., Granja, P. L., Fricain, J.-C., Bidarra, S. J., Renard, M., Bareille, R., ... Barbosa, M. A. (2009). The effect of the co-immobilization of human osteoprogenitors and endothelial cells within alginate microspheres on mineralization in a bone defect. Biomaterials, 30(19), 3271-3278.

Groll, J., Boland, T., Blunk, T., Burdick, J. A., Cho, D.-W., Dalton, P. D., ... Mironov, V. A (2016). Biofabrication: Reappraising the definition of an evolving field. Biofabrication, 8(1), 013001

Gross, K. A., \& Rodríguez-Lorenzo, L. M. (2004). Biodegradable composite scaffolds with an interconnected spherical network for bone tissue engineering. Biomaterials, 25(20), 4955-4962.

Gruskin, E., Doll, B. A., Futrell, F. W., Schmitz, J. P., \& Hollinger, J. O. (2012). Demineralized bone matrix in bone repair: History and use. Advanced Drug Delivery Reviews, 64(12), 1063-1077.

Guan, J., Liu, Q., Zhang, X., Zhang, Y., Chokshi, R., Wu, H., ... Mao, S. (2018). Alginate as a potential diphase solid dispersion carrier with enhanced drug dissolution and improved storage stability. European Journal of Pharmaceutical Sciences, 114, 346-355.

Hadef, I., Omri, M., Edwards-Lévy, F., \& Bliard, C. (2017). Influence of chemically modified alginate esters on the preparation of microparticles by transacylation with protein in W/O emulsions. Carbohydrate Polymers, 157, 275-281.

Henkel, J., Woodruff, M. A., Epari, D. R., Steck, R., Glatt, V., Dickinson, I. C., .. Hutmacher, D. W. (2013). Bone regeneration based on tissue engineering conceptions-A 21st century perspective. Bone Research, 1, 216-248.

Hosoya, K., Ohtsuki, C., Kawai, T., Kamitakahara, M., Ogata, S., Miyazaki, T., ... Tanihara, M. (2004). A novel covalently crosslinked gel of alginate and silane with the ability to form bone-like apatite. Journal of Biomedical Materials Research Part A, 71(4), $596-601$.

Hu, J., Hou, Y., Park, H., Choi, B., Hou, S., Chung, A., ... Lee, M. (2012). Visible light crosslinkable chitosan hydrogels for tissue engineering. Acta Biomaterialia, 8(5), 1730-1738.

Imeson, A. (2010). Food stabilisers, thickeners and gelling agents. Wiley Online Library.

Ishikawa, K., Miyamoto, Y., Kon, M., Nagayama, M., \& Asaoka, K. (1995). Non-decay type fast-setting calcium phosphate cement: Composite with sodium alginate. Biomaterials, 16(7), 527-532.

Izadifar, Z., Chang, T., Kulyk, W., Chen, X., \& Eames, B. F. (2015). Analyzing biological performance of 3D-printed, cell-impregnated hybrid constructs for cartilage tissue engineering. Tissue Engineering Part C, Methods, 22(3), 173-188.

Jang, C. H., Ahn, S. H., Yang, G.-H., \& Kim, G. H. (2016). A MSCs-laden polycaprolactone/ collagen scaffold for bone tissue regeneration. RSC Advances, 6(8), 6259-6265.

Jang, T.-S., Jung, H.-D., Pan, H. M., Han, W. T., Chen, S., \& Song, J. (2018). 3D printing of hydrogel composite systems: Recent advances in technology for tissue engineering. International Journal of Bioprinting, 4(1).

Jayakumar, R., Prabaharan, M., Kumar, P. S., Nair, S., \& Tamura, H. (2011). Biomaterials based on chitin and chitosan in wound dressing applications. Biotechnology Advances, 29(3), 322-337.

Jeon, O., Alt, D. S., Ahmed, S. M., \& Alsberg, E. (2012). The effect of oxidation on the degradation of photocrosslinkable alginate hydrogels. Biomaterials, 33(13), 3503-3514.

Jeon, O., Bouhadir, K. H., Mansour, J. M., \& Alsberg, E. (2009). Photocrosslinked alginate hydrogels with tunable biodegradation rates and mechanical properties. Biomaterials, 30(14), 2724-2734.

Jewad, R., Bentham, C., Hancock, B., Bonfield, W., \& Best, S. M. (2008). Dispersant selection for aqueous medium pressure injection moulding of anhydrous dicalcium phosphate. Journal of the European Ceramic Society, 28(3), 547-553.

Jia, J., Richards, D. J., Pollard, S., Tan, Y., Rodriguez, J., Visconti, R. P., ... Markwald, R. R. (2014). Engineering alginate as bioink for bioprinting. Acta Biomaterialia, 10(10), 4323-4331.

Jiménez-Gallegos, R., Rodríguez-Lorenzo, L. M., San Roman, J., \& Téllez-Jurado, L. (2017). Preparation, bioactivity, and cytotoxicity studies of poly (ester urethane) s/ SiO2 nanocomposites. Journal of Thermoplastic Composite Materials 0892705717744831.

Jin, Y., Liu, C., Chai, W., Compaan, A., \& Huang, Y. (2017). Self-supporting nanoclay as internal scaffold material for direct printing of Soft hydrogel composite structures in air. ACS Applied Materials \& Interfaces, 9(20), 17456-17465.

Jovic, T. H., Kungwengwe, G., Mills, A., \& Whitaker, I. S. (2019). Plant-derived biomaterials: A review of 3D bioprinting and biomedical applications. Frontiers of Mechanical Engineering, 5, 19.

Kang, H.-W., Lee, S. J., Ko, I. K., Kengla, C., Yoo, J. J., \& Atala, A. (2016). A 3D bioprinting system to produce human-scale tissue constructs with structural integrity. Nature Biotechnology, 34(3), 312.

Karageorgiou, V., \& Kaplan, D. (2005). Porosity of 3D biomaterial scaffolds and osteogenesis. Biomaterials, 26(27), 5474-5491.

Kelishomi, Z. H., Goliaei, B., Mahdavi, H., Nikoofar, A., Rahimi, M., Moosavi-Movahedi, A. A., ... Bigdeli, B. (2016). Antioxidant activity of low molecular weight alginate produced by thermal treatment. Food Chemistry, 196, 897-902.

Kim, W. S., Mooney, D. J., Arany, P. R., Lee, K., Huebsch, N., \& Kim, J. (2012). Adipose 
tissue engineering using injectable, oxidized alginate hydrogels. Tissue Engineering Part A, 18(7-8), 737-743.

Klöck, G., Pfeffermann, A., Ryser, C., Gröhn, P., Kuttler, B., Hahn, H.-J., ... Zimmermann, U. (1997). Biocompatibility of mannuronic acid-rich alginates. Biomaterials, 18(10), 707-713.

Kolk, A., Handschel, J., Drescher, W., Rothamel, D., Kloss, F., Blessmann, M., ... Smeets, R. (2012). Current trends and future perspectives of bone substitute materials-from space holders to innovative biomaterials. Journal of Cranio-Maxillofacial Surgery, 40(8), 706-718.

Kong, H. J., Alsberg, E., Kaigler, D., Lee, K. Y., \& Mooney, D. J. (2004). Controlling degradation of hydrogels via the size of crosslinked junctions. Advanced Materials, 16(21), 1917-1921.

Krishnan, L., Priddy, L. B., Esancy, C., Li, M.-T. A., Stevens, H. Y., Jiang, X., ... Guldberg, R. E. (2015). Hydrogel-based delivery of rhBMP-2 improves healing of large bone defects compared with autograft. Clinical Orthopaedics and Related Research, 473(9) $2885-2897$.

Küçüktürkmen, B., Öz, U. C., \& Bozkir, A. (2017). In situ hydrogel formulation for intraarticular application of diclofenac sodium-loaded polymeric nanoparticles. Turkish Journal of Pharmaceutical Sciences, 14(1).

Kundu, J., Shim, J. H., Jang, J., Kim, S. W., \& Cho, D. W. (2015). An additive manufacturing-based PCL-alginate-chondrocyte bioprinted scaffold for cartilage tissue engineering. Journal of Tissue Engineering and Regenerative Medicine, 9(11), $1286-1297$.

Kuo, C. K., \& Ma, P. X. (2001). Ionically crosslinked alginate hydrogels as scaffolds for tissue engineering: Part 1. Structure, gelation rate and mechanical properties. Biomaterials, 22(6), 511-521.

Kurczewska, J., Cegłowski, M., Pecyna, P., Ratajczak, M., Gajęcka, M., \& Schroeder, G. (2017). Molecularly imprinted polymer as drug delivery carrier in alginate dressing Materials Letters, 201, 46-49.

Lee, A., Langford, C. R., Rodriguez-Lorenzo, L. M., Thissen, H., \& Cameron, N. R. (2017). Bioceramic nanocomposite thiol-acrylate polyHIPE scaffolds for enhanced osteoblastic cell culture in 3D. Biomaterials Science, 5(10), 2035-2047.

Lee, G.-S., Park, J.-H., Won, J.-E., Shin, U. S., \& Kim, H.-W. (2011). Alginate combined calcium phosphate cements: Mechanical properties and in vitro rat bone marrow stromal cell responses. Journal of Materials Science Materials in Medicine, 22(5), 1257-1268.

Lee, H. J., Choi, H. W., Kim, K. J., \& Lee, S. C. (2006). Modification of hydroxyapatite nanosurfaces for enhanced colloidal stability and improved interfacial adhesion in nanocomposites. Chemistry of Materials, 18(21), 5111-5118.

Lee, J. M., Sing, S. L., Zhou, M., \& Yeong, W. Y. (2018). 3D bioprinting processes: A perspective on classification and terminology. International Journal of Bioprinting, 4(2).

Lee, K. Y., \& Mooney, D. J. (2012). Alginate: Properties and biomedical applications Progress in Polymer Science, 37(1), 106-126.

Lee, K. Y., Rowley, J. A., Eiselt, P., Moy, E. M., Bouhadir, K. H., \& Mooney, D. J. (2000). Controlling mechanical and swelling properties of alginate hydrogels independently by cross-linker type and cross-linking density. Macromolecules, 33(11), 4291-4294.

Lee, Y. H., Lee, B. W., Jung, Y. C., Yoon, B. I., Woo, H. M., \& Kang, B. J. (2018) Application of alginate microbeads as a carrier of bone morphogenetic protein-2 for bone regeneration. Journal of Biomedical Materials Research Part B, Applied Biomaterials.

Leijs, M., van Buul, G., Nieboer, M., Haeck, J., Kops, N., Bos, P., ... van Osch, G. (2016). Endurable injectable mesenchymal stem cell therapy for osteoarthritis by encapsulation in alginate constructs. Osteoarthritis and Cartilage, 24, S12-S13.

Lewandowska-Łańcucka, J., Mystek, K., Mignon, A., Van Vlierberghe, S., Łatkiewicz, A., \& Nowakowska, M. (2017). Alginate-and gelatin-based bioactive photocross-linkable hybrid materials for bone tissue engineering. Carbohydrate Polymers, 157, 1714-1722.

Li, P., Ohtsuki, C., Kokubo, T., Nakanishi, K., Soga, N., Nakamura, T., ... Yamamuro, T. (1992). Apatite formation induced by silica gel in a simulated body fluid. Journal of the American Ceramic Society, 75(8), 2094-2097.

Li, X., Weng, Y., Kong, X., Zhang, B., Li, M., Diao, K., ... Chen, H. (2012). A covalently crosslinked polysaccharide hydrogel for potential applications in drug delivery and tissue engineering. Journal of Materials Science Materials in Medicine, 23(12), $2857-2865$.

Li, X., Xu, A., Xie, H., Yu, W., Xie, W., \& Ma, X. (2010). Preparation of low molecular weight alginate by hydrogen peroxide depolymerization for tissue engineering Carbohydrate Polymers, 79(3), 660-664.

Lin, C.-C., \& Anseth, K. S. (2009). PEG hydrogels for the controlled release of biomolecules in regenerative medicine. Pharmaceutical Research, 26(3), 631-643.

Lin, H. R., \& Yeh, Y. J. (2004). Porous alginate/hydroxyapatite composite scaffolds for bone tissue engineering: Preparation, characterization, and in vitro studies. Journal of Biomedical Materials Research Part B, Applied Biomaterials, 71(1), 52-65.

Lisby, S., Gniadecki, R., \& Wulf, H. C. (2005). UV-induced DNA damage in human keratinocytes: Quantitation and correlation with long-term survival. Experimental Dermatology, 14(5), 349-355.

Liu, F., Li, R., Mao, L., \& Gao, Y. (2018). Ethanol-induced composite hydrogel based on propylene glycol alginate and zein: Formation, characterization and application. Food Chemistry, 255, 390-398.

Liu, M., Zeng, X., Ma, C., Yi, H., Ali, Z., Mou, X., ... He, N. (2017). Injectable hydrogels for cartilage and bone tissue engineering. Bone Research, 5, 17014.

Liu, S., Hu, Y., Zhang, J., Bao, S., Xian, L., Dong, X., ... Zhou, W. (2019). Bioactive and biocompatible macroporous scaffolds with tunable performances prepared based on 3D printing of the pre-crosslinked sodium alginate/hydroxyapatite hydrogel ink. Macromolecular Materials and Engineering, 304(4), 1800698.

Luo, Y., Wu, C., Lode, A., \& Gelinsky, M. (2012). Hierarchical mesoporous bioactive glass/ alginate composite scaffolds fabricated by three-dimensional plotting for bone tissue engineering. Biofabrication, 5(1), 015005.

Majmudar, G., Bole, D., Goldstein, S. A., \& Bonadio, J. (1991). Bone cell culture in a three-dimensional polymer bead stabilizes the differentiated phenotype and provides evidence that osteoblastic cells synthesize type III collagen and fibronectin. Journal of Bone and Mineral Research, 6(8), 869-881.

Malda, J., Visser, J., Melchels, F. P., Jüngst, T., Hennink, W. E., Dhert, W. J., .. Hutmacher, D. W. (2013). 25th anniversary article: Engineering hydrogels for biofabrication. Advanced Materials, 25(36), 5011-5028.

Man, Y., Wang, P., Guo, Y., Xiang, L., Yang, Y., Qu, Y., ... Deng, L. (2012). Angiogenic and osteogenic potential of platelet-rich plasma and adipose-derived stem cell laden alginate microspheres. Biomaterials, 33(34), 8802-8811.

Markstedt, K., Mantas, A., Tournier, I., Martínez Ávila, H.c., Hägg, D., \& Gatenholm, P. (2015). 3D bioprinting human chondrocytes with nanocellulose-alginate bioink for cartilage tissue engineering applications. Biomacromolecules, 16(5), 1489-1496.

Markusen, J. F., Mason, C., Hull, D. A., Town, M. A., Tabor, A. B., Clements, M., ... Pete (2006). Behavior of adult human mesenchymal stem cells entrapped in alginateGRGDY beads. Tissue Engineering, 12(4), 821-830.

Marsich, E., Bellomo, F., Turco, G., Travan, A., Donati, I., \& Paoletti, S. (2013). Nanocomposite scaffolds for bone tissue engineering containing silver nanoparticles: Preparation, characterization and biological properties. Journal of Materials Science Materials in Medicine, 24(7), 1799-1807.

Meillisa, A., Woo, H.-C., \& Chun, B.-S. (2015). Production of monosaccharides and bioactive compounds derived from marine polysaccharides using subcritical water hydrolysis. Food Chemistry, 171, 70-77.

Morais, D., Rodrigues, M., Lopes, M., Coelho, M., Maurício, A. C., Gomes, R., ... Botelho, C. (2013). Biological evaluation of alginate-based hydrogels, with antimicrobial features by Ce (III) incorporation, as vehicles for a bone substitute. Journal of Materials Science Materials in Medicine, 24(9), 2145-2155.

Moroni, L., Boland, T., Burdick, J. A., De Maria, C., Derby, B., Forgacs, G., ... Mironov, V. A. (2017). Biofabrication: A guide to technology and terminology. Trends in Biotechnology.

Moshaverinia, A., Chen, C., Akiyama, K., Ansari, S., Xu, X., Chee, W. W., ... Shi, S. (2012). Alginate hydrogel as a promising scaffold for dental-derived stem cells: An in vitro study. Journal of Materials Science Materials in Medicine, 23(12), 3041-3051.

Oliveira, S. M., Barrias, C. C., Almeida, I. F., Costa, P. C., Ferreira, M. R. P., Bahia, M. F., .. Barbosa, M. A. (2008). Injectability of a bone filler system based on hydroxyapatite microspheres and a vehicle with in situ gel-forming ability. Journal of Biomedical Materials Research Part B, Applied Biomaterials, 87(1), 49-58.

Osmokrovic, A., Jancic, I., Vunduk, J., Petrovic, P., Milenkovic, M., \& Obradovic, B. (2018). Achieving high antimicrobial activity: Composite alginate hydrogel beads releasing activated charcoal with an immobilized active agent. Carbohydrate Polymers, 196, 279-288.

Otterlei, M., Ostgaard, K., Skjåk-Bræk, G., Smidsrød, O., Soon-Shiong, P., \& Espevik, T. (1991). Induction of cytokine production from human monocytes stimulated with alginate. Journal of Immunotherapy, 10(4), 286-291.

Ozbolat, I. T., \& Hospodiuk, M. (2016). Current advances and future perspectives in extrusion-based bioprinting. Biomaterials, 76, 321-343.

Painter, T., \& Larsen, B. R. (1970). Formation of hemiacetals between neighbouring hexuronic acid residues during the periodate oxidation of alginate. Acta Chemica Scandinavica, 24(3), 813-833.

Palmer, L. C., Newcomb, C. J., Kaltz, S. R., Spoerke, E. D., \& Stupp, S. I. (2008). Biomimetic systems for hydroxyapatite mineralization inspired by bone and enamel Chemical Reviews, 108(11), 4754-4783.

Park, H., Kang, S. W., Kim, B. S., Mooney, D. J., \& Lee, K. Y. (2009). Shear-reversibly crosslinked alginate hydrogels for tissue engineering. Macromolecular Bioscience, 9(9), 895-901.

Park, J., Lee, S. J., Chung, S., Lee, J. H., Kim, W. D., Lee, J. Y., ... Park, S. A. (2017). Cellladen 3D bioprinting hydrogel matrix depending on different compositions for soft tissue engineering: Characterization and evaluation. Materials Science and Engineering C, 71, 678-684.

Paxton, N., Smolan, W., Böck, T., Melchels, F., Groll, J., \& Jungst, T. (2017). Proposal to assess printability of bioinks for extrusion-based bioprinting and evaluation of rheological properties governing bioprintability. Biofabrication, 9(4), 044107.

Pereira, R. F., \& Bártolo, P. J. (2014). Photopolymerizable hydrogels in regenerative medicine and drug delivery. Future medicine.

Pereira, R. F., \& Bártolo, P. J. (2015). 3D bioprinting of photocrosslinkable hydrogel constructs. Journal of Applied Polymer Science, 132(48).

Perez, R. A., \& Kim, H. W. (2013). Core-shell designed scaffolds of alginate/alphatricalcium phosphate for the loading and delivery of biological proteins. Journal of Biomedical Materials Research Part A, 101(4), 1103-1112.

Pfeifer, G. P., You, Y.-H., \& Besaratinia, A. (2005). Mutations induced by ultraviolet light. Mutation Research/Fundamental and Molecular Mechanisms of Mutagenesis, 571(1-2), 19-31.

Pina, S., Oliveira, J. M., \& Reis, R. L. (2015). Natural-based nanocomposites for bone tissue engineering and regenerative medicine: A review. Advanced Materials, 27(7), 1143-1169.

Plaza, J. Q., Garzón, L. B., Gimenez, B. B., Moraleda, B. F.-M., Collía, F., \& RodríguezLorenzo, L. M. (2016). Application of calcium phosphates and fibronectin as complementary treatment for osteoporotic bone fractures. Injury, 47, S15-S21.

Qi, M., Mørch, Y., Lacík, I., Formo, K., Marchese, E., Wang, Y., ... Barbaro, B. (2012). Survival of human islets in microbeads containing high guluronic acid alginate crosslinked with $\mathrm{Ca} 2+$ and Ba2 +. Xenotransplantation, 19(6), 355-364.

Qiu, M., Chen, D., Shen, C., Shen, J., Zhao, H., \& He, Y. (2017). Preparation of in situ forming and injectable alginate/mesoporous Sr-containing calcium silicate composite cement for bone repair. RSC Advances, 7(38), 23671-23679. 
Radhakrishnan, J., Subramanian, A., Krishnan, U. M., \& Sethuraman, S. (2016). Injectable and 3D bioprinted polysaccharide hydrogels: From cartilage to osteochondral tissue engineering. Biomacromolecules, 18(1), 1-26.

Raja, N., \& Yun, H.-s. (2016). A simultaneous 3D printing process for the fabrication of bioceramic and cell-laden hydrogel core/shell scaffolds with potential application in bone tissue regeneration. Journal of Materials Chemistry B, 4(27), 4707-4716.

Reakasame, S., \& Boccaccini, A. R. (2017). Oxidized alginate-based hydrogels for tissue engineering applications: A review. Biomacromolecules, 19(1), 3-21.

Rescignano, N., Hernandez, R., Lopez, L. D., Calvillo, I., Kenny, J. M., \& Mijangos, C. (2016). Preparation of alginate hydrogels containing silver nanoparticles: A facile approach for antibacterial applications. Polymer International, 65(8), 921-926.

Rezvanain, M., Ahmad, N., Amin, M. C. I. M., \& Ng, S.-F. (2017). Optimization, characterization, and in vitro assessment of alginate-pectin ionic cross-linked hydrogel film for wound dressing applications. International Journal of Biological Macromolecules, 97, 131-140.

Rezvanian, M., Amin, M. C. I. M., \& Ng, S.-F. (2016). Development and physicochemical characterization of alginate composite film loaded with simvastatin as a potential wound dressing. Carbohydrate Polymers, 137, 295-304.

Rhim, J.-W. (2004). Physical and mechanical properties of water resistant sodium alginate films. LWT-Food science and technology, 37(3), 323-330.

Ribeiro, A., Blokzijl, M. M., Levato, R., Visser, C. W., Castilho, M., Hennink, W. E., .. Malda, J. (2017). Assessing bioink shape fidelity to aid material development in 3D bioprinting. Biofabrication, 10(1), 014102.

Rodríguez-Lorenzo, L. M., García-Carrodeguas, R., Rodríguez, M. A., De Aza, S., Jiménez, J., López-Bravo, A., ... Román, J. S. (2009). Synthesis, characterization, bioactivity and biocompatibility of nanostructured materials based on the wollastonite-poly (ethylmethacrylate-co-vinylpyrrolidone) system. Journal of Biomedical Materials Research Part A, 88(1), 53-64.

Roopavath, U. K., Soni, R., Mahanta, U., Deshpande, A. S., \& Rath, S. N. (2019). 3D printable $\mathrm{SiO} 2$ nanoparticle ink for patient specific bone regeneration. RSC Advances, 9(41), 23832-23842.

Roseti, L., Parisi, V., Petretta, M., Cavallo, C., Desando, G., Bartolotti, I., ... Grigolo, B. (2017). Scaffolds for bone tissue engineering: State of the art and new perspectives. Materials Science and Engineering C, 78, 1246-1262.

Rowley, J. A., Madlambayan, G., \& Mooney, D. J. (1999). Alginate hydrogels as synthetic extracellular matrix materials. Biomaterials, 20(1), 45-53.

Ruan, Q., Zhang, Y., Yang, X., Nutt, S., \& Moradian-Oldak, J. (2013). An amelogenin-chitosan matrix promotes assembly of an enamel-like layer with a dense interface. Acta Biomaterialia, 9(7), 7289-7297.

Russo, L., Taraballi, F., Lupo, C., Poveda, A., Jiménez-Barbero, J., Sandri, M., ... Cipolla, L. (2014). Carbonate hydroxyapatite functionalization: A comparative study towards (bio) molecules fixation. Interface Focus, 4(1), 20130040.

Sakai, S., Kamei, H., Mori, T., Hotta, T., Ohi, H., Nakahata, M., ... Taya, M. (2018). Visible light-induced hydrogelation of an alginate derivative and application to stereolithographic bioprinting using a visible light projector and acid red. Biomacromolecules, 19(2), 672-679.

Sánchez-Téllez, D., Tellez-Jurado, L., \& Chavez-Alcala, J. F. (2014). Bioactivity and degradability of hybrids nano-composites materials with great application as bone tissue substitutes. Journal of Alloys and Compounds, 615, S670-S675.

Sánchez-Téllez, D., Tellez-Jurado, L., \& Rodríguez-Lorenzo, L. (2015). Optimization of the $\mathrm{CaO}$ and $\mathrm{P} 2 \mathrm{O} 5$ contents on PDMS-SiO2-CaO-P2O5 hybrids intended for bone regeneration. Journal of Materials Science, 50(18), 5993-6006.

Sánchez-Téllez, D., Téllez-Jurado, L., Rodríguez-Lorenzo, L., Mazo, M., Rubio, J., \& Tamayo, A. (2017). Surface effects on the degradation mechanism of bioactive PDMSSiO2-CaO-P2O5 hybrid materials intended for bone regeneration. Ceramics International, 43(1), 476-483.

Sarker, A., Amirian, J., Min, Y. K., \& Lee, B. T. (2015). HAp granules encapsulated oxidized alginate-gelatin-biphasic calcium phosphate hydrogel for bone regeneration. International Journal of Biological Macromolecules, 81, 898-911.

Sarker, B., Li, W., Zheng, K., Detsch, R., \& Boccaccini, A. R. (2016). Designing porous bone tissue engineering scaffolds with enhanced mechanical properties from composite hydrogels composed of modified alginate, gelatin, and bioactive glass. ACS Biomaterials Science \& Engineering, 2(12), 2240-2254.

Sarker, B., Zehnder, T., Rath, S. N., Horch, R. E., Kneser, U., Detsch, R., ... Boccaccini, A. R. (2017). Oxidized alginate-gelatin hydrogel: A favorable matrix for growth and osteogenic differentiation of adipose-derived stem cells in 3d. ACS Biomaterial Science \& Engineering, 3(8), 1730-1737.

Sarker, B. P., Dimitrios, G., Silva, R., Zehnder, T., Gul-E-Noor, F., Bertmer, M., ... Aldo, R. (2014). Fabrication of alginate-gelatin crosslinked hydrogel microcapsules and evaluation of the microstructure and physico-chemical properties. Journal of Materials Chemistry B, 2(11), 1470-1482.

Schloßmacher, U., Schröder, H. C., Wang, X., Feng, Q., Diehl-Seifert, B., Neumann, S., ... Müller, W. E. (2013). Alginate/silica composite hydrogel as a potential morphogenetically active scaffold for three-dimensional tissue engineering. RSC Advances, 3(28), 11185-11194.

Schütz, K., Placht, A. M., Paul, B., Brüggemeier, S., Gelinsky, M., \& Lode, A. (2017). Three-dimensional plotting of a cell-laden alginate/methylcellulose blend: Towards biofabrication of tissue engineering constructs with clinically relevant dimensions. Journal of Tissue Engineering and Regenerative Medicine, 11(5), 1574-1587.

Segredo-Morales, E., García-García, P., Reyes, R., Pérez-Herrero, E., Delgado, A., \& Évora, C. (2018). Bone regeneration in osteoporosis by delivery BMP-2 and PRGF from Tetronic-Alginate composite thermogel. International Journal of Pharmaceutics.

Silberman, F. S., \& Varaona, O. (2011). Ortopedia y Traumatologia/Orthopedics and Traumatology. Ed. Médica Panamericana.

Smidsrød, O., Glover, R., \& Whittington, S. G. (1973). The relative extension of alginates having different chemical composition. Carbohydrate Research, 27(1), 107-118.
Sowjanya, J., Singh, J., Mohita, T., Sarvanan, S., Moorthi, A., Srinivasan, N., .. Selvamurugan, N. (2013). Biocomposite scaffolds containing chitosan/alginate/nanosilica for bone tissue engineering. Colloids and Surfaces B: Biointerfaces, 109, 294-300.

Stintzing, F. C., \& Carle, R. (2005). Cactus stems (Opuntia spp.): A review on their chemistry, technology, and uses. Molecular Nutrition \& Food Research, 49(2), 175-194.

Stockwell, A., Davis, S., \& Walker, S. (1986). In vitro evaluation of alginate gel systems as sustained release drug delivery systems. Journal of Controlled Release, 3(1-4), $167-175$.

Stokke, B. T., Smidsrød, O., \& Brant, D. A. (1993). Predicted influence of monomer sequence distribution and acetylation on the extension of naturally occurring alginates. Carbohydrate Polymers, 22(1), 57-66.

Straccia, M. C., d'Ayala, G. G., Romano, I., \& Laurienzo, P. (2015). Novel zinc alginate hydrogels prepared by internal setting method with intrinsic antibacterial activity. Carbohydrate Polymers, 125, 103-112.

Su, W.-R., Liu, H.-Y., \& Yeh, M.-1. (2017). Effect of injectable alpha calcium sulfate hemihydrate for repair of Hill-Sachs lesion in a rabbit model. Asia-Pacific Journal of Sports Medicine Arthroscopy Rehabilitation and Technology, 9, 121.

Sun, J., \& Tan, H. (2013). Alginate-based biomaterials for regenerative medicine applications. Materials, 6(4), 1285-1309.

Szekalska, M., Puciłowska, A., Szymańska, E., Ciosek, P., \& Winnicka, K. (2016). Alginate: Current use and future perspectives in pharmaceutical and biomedical applications. International Journal of Polymer Science, 2016.

Tan, H., \& Marra, K. G. (2010). Injectable, biodegradable hydrogels for tissue engineering applications. Materials, 3(3), 1746-1767.

Tønnesen, H. H., \& Karlsen, J. (2002). Alginate in drug delivery systems. Drug Development and Industrial Pharmacy, 28(6), 621-630.

Valentin, T. M., Leggett, S. E., Chen, P.-Y., Sodhi, J. K., Stephens, L. H., McClintock, H. D., Wong, I. Y. (2017). Stereolithographic printing of ionically-crosslinked alginate hydrogels for degradable biomaterials and microfluidics. Lab on a Chip, 17(20), 3474-3488.

Vo, T., Shah, S., Lu, S., Tatara, A., Lee, E., Roh, T., ... Mikos, A. (2016). Injectable dualgelling cell-laden composite hydrogels for bone tissue engineering. Biomaterials, 83, $1-11$.

Vold, I. M. N., Kristiansen, K. A., \& Christensen, B. E. (2006). A study of the chain stiffnes and extension of alginates, in vitro epimerized alginates, and periodate-oxidized alginates using size-exclusion chromatography combined with light scattering and viscosity detectors. Biomacromolecules, 7(7), 2136-2146.

Wang, Q., Wang, Q., \& Teng, W. (2016). Injectable, degradable, electroactive nanocomposite hydrogels containing conductive polymer nanoparticles for biomedical applications. International Journal of Nanomedicine, 11, 131.

Wang, X., Jiang, M., Zhou, Z., Gou, J., \& Hui, D. (2017). 3D printing of polymer matrix composites: A review and prospective. Composites Part B Engineering, 110, 442-458.

Wang, X., Tolba, E., Schröder, H. C., Neufurth, M., Feng, Q., Diehl-Seifert, B., ... Müller, W. E. (2014). Effect of bioglass on growth and biomineralization of SaOS-2 cells in hydrogel after 3D cell bioprinting. PLoS One, 9(11), e112497.

Wei, Z., Yang, J. H., Liu, Z. Q., Xu, F., Zhou, J. X., Zrínyi, M., ... Chen, Y. M. (2015). Novel biocompatible polysaccharide-based self-healing hydrogel. Advanced Functional Materials, 25(9), 1352-1359.

Welsch, U., \& Sobotta, J. (2008). Histología. Ed. Médica Panamericana.

Whittington, S. G. (1971). Conformational energy calculations on alginic acid I. Helix parameters and flexibility of the homopolymers. Biopolymers, 10(9), 1481-1489.

Wright, B., De Bank, P. A., Luetchford, K. A., Acosta, F. R., \& Connon, C. J. (2014). Oxidized alginate hydrogels as niche environments for corneal epithelial cells. Journal of Biomedical Materials Research Part A, 102(10), 3393-3400.

Wu, Y., Yuan, L., Sheng, N.-a., Gu, Z.-q., Feng, W.-h., Yin, H.-y., ... Mo, X.-m. (2017). A soft tissue adhesive based on aldehyde-sodium alginate and amino-carboxymethyl chitosan preparation through the Schiff reaction. Frontiers of Materials Science, 11(3), 215-222.

Xiong, R., Zhang, Z., \& Huang, Y. (2015). Identification of optimal printing conditions for laser printing of alginate tubular constructs. Journal of Manufacturing Processes, 20, $450-455$.

Xu, C., Wang, X., Zhou, J., Huan, Z., \& Chang, J. (2018). Bioactive tricalcium silicate/ alginate composite bone cements with enhanced physicochemical properties. Journal of Biomedical Materials Research Part B, Applied Biomaterials, 106(1), 237-244.

Xu, M., Feng, C., Wang, J., Lang, X., Xia, G., Yu, X., ... Liu, Y. (2017). In vitro heterogeneous degradation of alginate and its validation of different molecular weight on blood bio-compatibility. Journal of Biomaterials Science Polymer Edition, 28(4), 380-393.

Xu, X., Bi, D., \& Wan, M. (2016). Characterization and immunological evaluation of low molecular-weight alginate derivatives. Current Topics in Medicinal Chemistry, 16(8), 874-887.

Xu, Z., \& Lam, M. T. (2018). Alginate application for heart and cardiovascular diseases. Alginates and their biomedical applications. Springer185-212.

Yan, H., Chen, X., Feng, M., Shi, Z., Zhang, D., \& Lin, Q. (2017). Layer-by-layer assembly of $3 \mathrm{D}$ alginate-chitosan-gelatin composite scaffold incorporating bacterial cellulose nanocrystals for bone tissue engineering. Materials Letters, 209, 492-496.

Yan, J., Huang, Y., \& Chrisey, D. B. (2012). Laser-assisted printing of alginate long tubes and annular constructs. Biofabrication, 5(1), 015002.

Yang, J.-A. Y., Junseok, Hwang, B. W., Hoffman, A. S., \& Hahn, S. K. (2014). In situforming injectable hydrogels for regenerative medicine. Progress in Polymer Science, 39(12), 1973-1986.

Yang, J.-S., Xie, Y.-J., \& He, W. (2011). Research progress on chemical modification of alginate: A review. Carbohydrate Polymers, 84(1), 33-39.

Yoon, S., Park, J. A., Lee, H. R., Yoon, W. H., Hwang, D. S., \& Jung, S. (2018). Inkjet-spray hybrid printing for 3D freeform fabrication of multilayered hydrogel structures. Advanced Healthcare Materials1800050. 
You, F., Chen, D. X., Cooper, D. M., Chang, T., \& Eames, F. B. (2018). Homogeneous hydroxyapatite/alginate composite hydrogel promotes calcified cartilage matrix deposition with potential for three-dimensional bioprinting. Biofabrication.

Yuan, L., Wu, Y., Gu, Q.-s., El-Hamshary, H., El-Newehy, M., \& Mo, X. (2017). Injectable photo crosslinked enhanced double-network hydrogels from modified sodium alginate and gelatin. International Journal of Biological Macromolecules, 96, 569-577.

Zhang, L. F., Yang, D. J., Chen, H. C., Sun, R., Xu, L., Xiong, Z. C., ... Xiong, C. D. (2008). An ionically crosslinked hydrogel containing vancomycin coating on a porous scaffold for drug delivery and cell culture. International Journal of Pharmaceutics, 353(1), 74-87.
Zhao, X., Huebsch, N., Mooney, D. J., \& Suo, Z. (2010). Stress-relaxation behavior in gels with ionic and covalent crosslinks. Journal of Applied Physics, 107(6), 063509.

Zhou, H., \& Xu, H. H. (2011). The fast release of stem cells from alginate-fibrin microbeads in injectable scaffolds for bone tissue engineering. Biomaterials, 32(30), 7503-7513.

Zhu, K., Chen, N., Liu, X., Mu, X., Zhang, W., Wang, C., ... Zhang, Y. S. (2018). A general strategy for extrusion bioprinting of bio-macromolecular bioinks through alginate-templated dual-stage crosslinking. Macromolecular Bioscience, 18(9), 1800127.

Zhu, W., Ma, X., Gou, M., Mei, D., Zhang, K., \& Chen, S. (2016). 3D printing of functional biomaterials for tissue engineering. Current Opinion in Biotechnology, 40, 103-112. 